\title{
Miniaturization of fluorescence sensing in optofluidic devices
}

\author{
Daniel Măriuţa ${ }^{1,2} \cdot$ Stéphane Colin ${ }^{2} \cdot$ Christine Barrot-Lattes $^{2} \cdot$ Stéphane Le Calvé $^{3,4}$ • Jan G. Korvink ${ }^{1}$. \\ Lucien Baldas ${ }^{2}$. Jürgen J. Brandner ${ }^{1}$ (B)
}

Received: 14 February 2020 / Accepted: 20 July 2020 / Published online: 3 August 2020

(c) The Author(s) 2020

\begin{abstract}
Successful development of a micro-total-analysis system ( $\mu$ TAS, lab-on-a-chip) is strictly related to the degree of miniaturization, integration, autonomy, sensitivity, selectivity, and repeatability of its detector. Fluorescence sensing is an optical detection method used for a large variety of biological and chemical assays, and its full integration within lab-on-a-chip devices remains a challenge. Important achievements were reported during the last few years, including improvements of previously reported methodologies, as well as new integration strategies. However, a universal paradigm remains elusive. This review considers achievements in the field of fluorescence sensing miniaturization, starting from off-chip approaches, representing miniaturized versions of their lab counter-parts, continuing gradually with strategies that aim to fully integrate fluorescence detection on-chip, and reporting the results around integration strategies based on optical-fiber-based designs, optical layer integrated designs, CMOS-based fluorescence sensing, and organic electronics. Further successful development in this field would enable the implementation of sensing networks in specific environments that, when coupled to Internetof-Things (IoT) and artificial intelligence (AI), could provide real-time data collection and, therefore, revolutionize fields like health, environmental, and industrial sensing.
\end{abstract}

Keywords Lab-on-a-chip · Off/on-chip integration strategy $\cdot$ Lab-on-a-CMOS $\cdot$ Microfluidic-PCB $\cdot$ In-plane optics · Organic electronics $\cdot$ Fluorescence detection

\section{Introduction}

Recent findings in the fields of microfluidics, integrated circuitry, microfabrication, and micromachining techniques have enabled considerable advancements in the miniaturized sensing technologies. Downscaling chemical and biological sensors result not only in ultra-portable devices, but in advantages such as enhanced process performance, higher analysis speed and reduced reagent consumption, significantly lowering fabrication, maintenance, and operational costs (Shakoor et al. 2018). Some fields that benefit from these achievements are healthcare monitoring (Boppart

1 Institute of Microstructure Technology, Karlsruhe Institute of Technology, Campus Nord, Hermann-von-Helmholtz-Platz 1, Eggenstein-Leopoldshafen, Germany

2 Institut Clément Ader (ICA), CNRS, INSA, ISAE-SUPAERO, Mines-Albi, UPS, Université de Toulouse, Toulouse, France

3 Group of Atmospheric Physical Chemistry, Institute of Chemistry and Processes for Energy, Environment and Health (ICPEES), University of Strasbourg, Strasbourg, France

4 In'Air Solutions, 25 rue Becquerel, Strasbourg, France

Jan G. Korvink

jan.korvink@kit.edu

Lucien Baldas

baldas@insa-toulouse.fr 
and Richards-Kortum 2014; Wessels and Raad 2016; Zi et al. 2016; Papageorgiou et al. 2017; Sharma et al. 2018), organ-on-a-chip (Zhang et al. 2015; Si Hadj Mohand et al. 2017; Kilic et al. 2018), drug screening (Zhang et al. 2015), food monitoring (Bhattacharya et al. 2017), and environment monitoring (Ricciardi et al. 2015a; Calvé et al. 2017; Rezende et al. 2019; Măriuța et al. 2020). Potential applications are countless with a huge impact on the quality of life (Chen et al. 2012). They are also directly linked with the next generation of smart cities (Zhao et al. 2015), artificial intelligence (AI), and Internet-of-Things (IoT).

Ideally, a lab-on-a-chip device should perform all the steps of a complete analysis in an integrated and automated fashion. This may include sampling, sample pre-treatment, chemical reactions, analytical separations, analyte detection, product isolation, and data analysis (Ríos et al. 2012). Even though the achievements reported in the literature are numerous, apparently the transition of these devices from lab prototyping to market is still limited, if not negligible (Volpatti and Yetisen 2014; Mohammed et al. 2015; Ackermann et al. 2016a), and the remaining challenges are still considerable. The main challenge is to identify and optimize strategies for the integration of all analysis functions into a cost-efficient, technician-free, robust microstructure, to develop fully-autonomous micro-analysis systems.

The integration of the detection mechanisms with microfluidics may be one of the most promising directions towards the widespread application of lab-on-a-chip devices (Wu and Gu 2011; Watts et al. 2012; Llobera et al. 2015). Generally, for detection within micro-integrated systems, mechanical, optical, and electrochemical methods are primarily used (Pires et al. 2014). The optical methods are usually preferred because they are robust, very sensitive, non-destructive, broadband and can be used for in-situ or in-line monitoring (Pires et al. 2014; Rodríguez-Ruiz et al. 2016; Yang and Gijs 2018). This combination of optics and microfluidics merged towards a relatively new field named optofluidics. Optofluidic systems aim to integrate the optical functions of detection in a single chip. The roadmap (Minzioni et al. 2017), the recent achievements (Chen et al. 2019), and the new perspectives (Song and Tan 2017) in the optofluidics field for lab-on-a-chip applications have also been published in (Zhu et al. 2013; Rodríguez-Ruiz et al. 2015; Zhang et al. 2016; Song et al. 2017). Among all-optical methods, fluorescence sensing is the most common analytical and diagnostic method in biological, chemical, and medical applications (Yang et al. 2015; Hong et al. 2017a; Wei et al. 2017), and it is largely accepted due to its capability to attain ultra-low detection limits (Ryu et al. 2011; Babikian et al. 2017).

Fluorescence is the property of a molecule to absorb light at a specific wavelength and emit it at a longer wavelength, a phenomenon known as the Stoke shift (see Fig. 1). Consequently, its quantification involves a light emission source (1) and a light detector (5). The amount of fluid involved in microfluidic systems is by definition reduced; therefore, the emitted fluorescence signal is weak. The ratio between fluorescence emission intensity and excitation beam intensity is defined as the quantum yield, which is molecule specific, usually the emission being three orders of magnitude lower than the excitation. This difference between excitation intensity and emitted fluorescence intensity makes the implementation of a complex optical path necessary, involving a system of lenses to focus (4) the fluorescence onto a photon detector (5) (Wei et al. 2017). Filters are used in traditional configurations of fluorescence detectors to selectively allow light with specific wavelengths. Emission filters (2) are used to reject parasitic components of the excitation light beam (1), allowing the passage of only the specific wavelength band needed for the excitation of the analyte (3). Detection filters (4) are used to stop scattered light from reaching the detector surface (5). The background noise levels are directly linked with the filtration efficiency. Noise is an undesirable
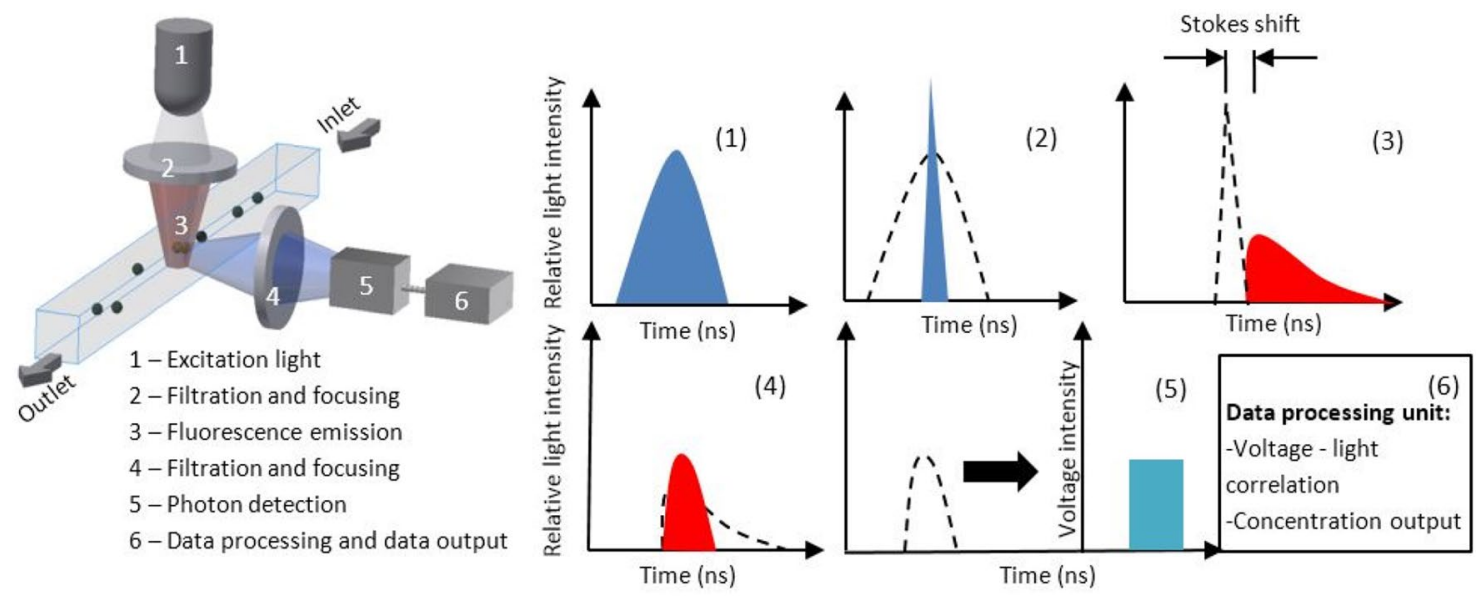

Fig. 1 General fluorescence detection scheme (orthogonal configuration) 
parameter quantifying the level of parasitic light reaching the detector. The signal-to-noise ratio (SNR) is used to characterize the performance of a detector, quantifying the ratio between fluorescence and parasitic light. This multitude of optical components make a system complex, bulky, and expensive (Hong et al. 2017a).

Thus, fluorescence sensing devices are not only sophisticated products integrating multidisciplinary know-how but also key components of lab-on-a-chip systems (Dandin et al. 2007). The miniaturization of fluorescence sensing devices is challenging —often developed systems end up being chipin-a-lab devices, instead of lab-on-a-chip platforms (Varsanik and Bernstein 2013; Shakoor et al. 2018). Different integration strategies of fluorescence optical detection have been reported during the last decade. Among them, the most promising one reviewed in this paper include optical-fiberbased designs, optical layer-integrated designs, the CMOS (complementary metal-oxide-semiconductor)-based microfluidic technologies, and organic electronics based designs. Efforts are continuously directed ahead to the identification of an integration paradigm that converges towards an ultraminiaturized fluorescence detection scheme (Shang and Zheng 2017). This system should assure the following characteristics: full working autonomy, multiplexing, low-cost fabrication, fast response time, robustness and compactness, specificity, and ultra-sensitivity (Pfeiffer and Nagl 2015). Even if nowadays the achievements are consistent, a generic strategy fulfilling all the above-mentioned specifications is still elusive. The main aim of this review is to identify the main recent integration strategies in the miniaturization of fluorescence detection systems, emphasizing design and manufacturing procedures, limits of detection achieved, and further possible improvements.

\section{Miniaturized off-chip fluorescence sensing architectures}

Off-chip approaches (or free-space designs) are usually miniaturized versions of conventional fluorescence detection systems (Babikian et al. 2017; Yang and Gijs 2018) generally being linked with the use of pinholes at the focal points along the optical path, to couple macro-scale optical detection to micro-scale detection volumes. In this case, light propagates in free-space before and after interacting with the target molecule, and optical elements (filters, lenses, mirrors, light sources, detectors) are separated from the microchip. Modular configuration confers them the advantage of convenient integration within a wide variety of already existing platforms, enabling them as plug-and-play microscopes (Zhang et al. 2015). Table 1 summarizes the achievements of some identified architectures.
The fluorescence microscope developed by Ghosh et al. (2011) (see Fig. 2. a) presented a high degree of miniaturization using state-of-the-art in the field of optoelectronics. It proposed an innovative design solution, embedding all optical elements within a $2.4 \mathrm{~cm}^{3}$ polyetheretherketone (PEEK) housing. A blue LED, integrated on a $6 \times 6 \mathrm{~mm}^{2}$ printed circuit board (PCB), was used as an excitation source. A drum lens was used to collect the emitted light, which was then passed through a $4 \times 4 \mathrm{~mm}^{2}$ excitation filter. A dichroic mirror directed the light to the sample, via a focusing gradient refractive index (GRIN) objective lens. The fluorescent emission passed through the objective lens, dichroic mirror, an emission filter, and an achromatic doublet lens, which in turn focused the image onto a CMOS sensor. The $640 \times 480$ pixel CMOS sensor, mounted on a $8.4 \times 8.4 \mathrm{~mm}^{2}$ printed circuit board had a $60 \%$ quantum efficiency at $530 \mathrm{~nm}$. The LED, image sensor, micro-lenses, and filters, were made using batch fabrication, decreasing the cost per unit. The device was built in an alignment-free configuration, but not stand-alone, requiring a computer for image processing. Data acquisition between microscope and computer was intermediated using an external PCB, allowing imaging at $35 \mathrm{~Hz}$ or $100 \mathrm{~Hz}$ over $300 \times 300$ pixels subregions.

More recently, other versions of miniaturized off-chip detectors were developed with fabrication costs per unit varying from $\$ 2000$ (Fang et al. 2016) to $\$ 10$ (Zhang et al. 2015). For example, a handheld fluorescence detector with a broad range of possible applications, compact size, and capability to work independently was proposed by (Fang et al. 2016) (see Fig. 2b). This device embedded a light source (450 nm laser diode), an optical circuit module (a $450 \mathrm{~nm}$ band-pass filter, a dichroic mirror, a collimating lens, a $525 \mathrm{~nm}$ band-pass filter, a $1.0 \mathrm{~mm}$ aperture), an optical detector (miniaturized photomultiplier tube), and an electronic module (signal recording, processing and displaying units). The stability was tested for more than $5 \mathrm{~h}$ for continuous detection of $100 \mathrm{nM}$ sodium fluorescein, and the relative standard deviation was below $1 \%$. Two configurations were tested, the quasi-confocal configuration was proven to be more advantageous compared to an orthogonal configuration, mainly from the perspective of possessing more top open space to be used for installing disposable interrogation cuvettes. The configuration used for positioning the optical elements is usually a compromise between the performance and the ease-of-use of the final device. A hand-held orthogonal detector that was proposed by Pan et al. (2018), where the fluorescence collection was performed at $45^{\circ}$, decreased the background scattering light intensity compared with a $90^{\circ}$ configuration. Compared to a confocal configuration, in the orthogonal configuration the excitation and collection paths were separated, leading to a reduced background signal without using complicated optical components. This 


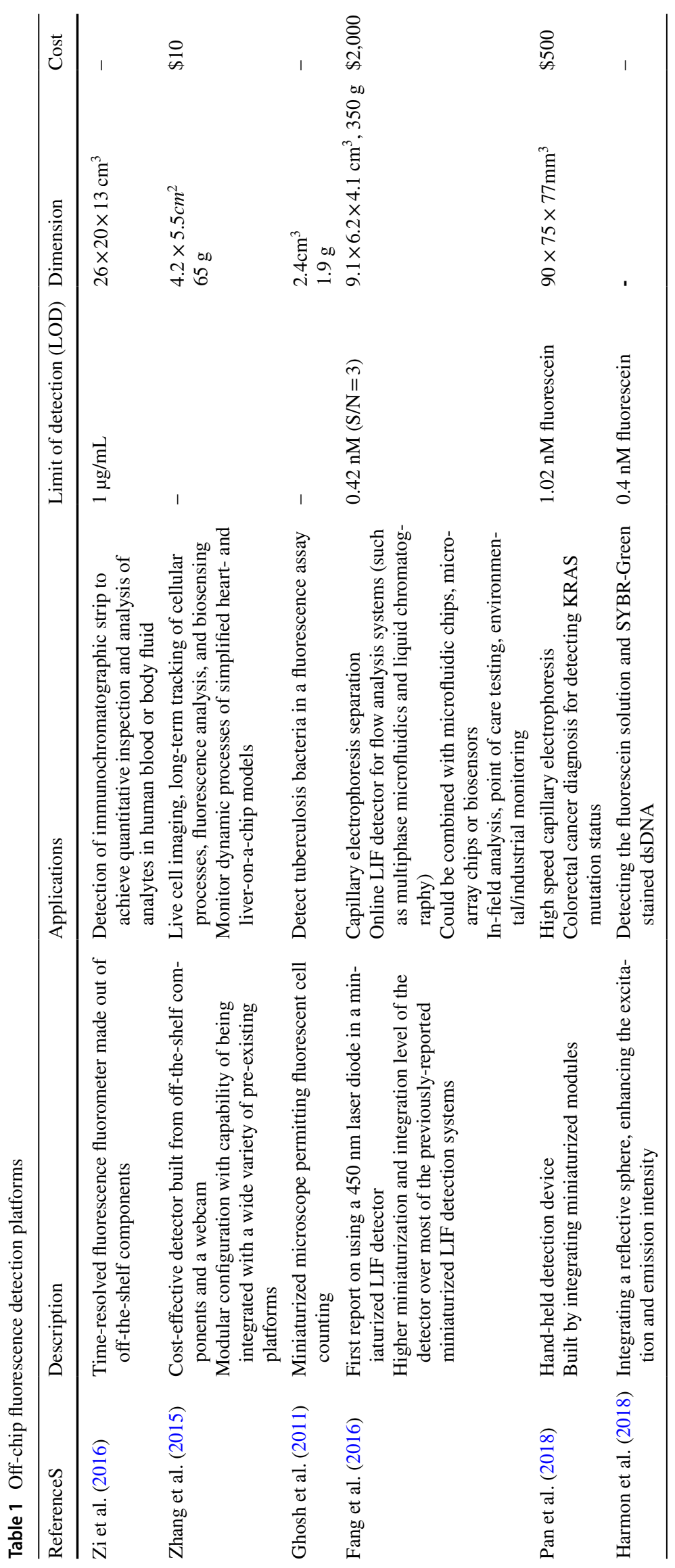




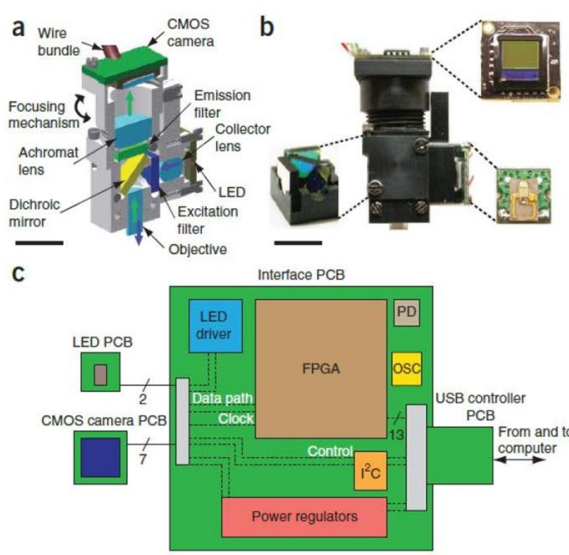

(a)

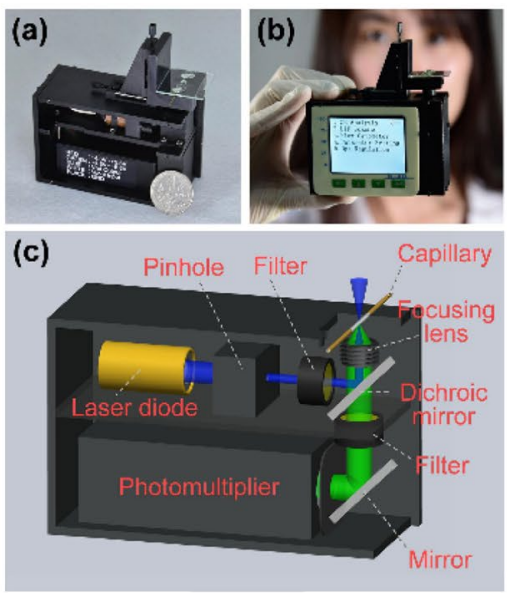

(b)

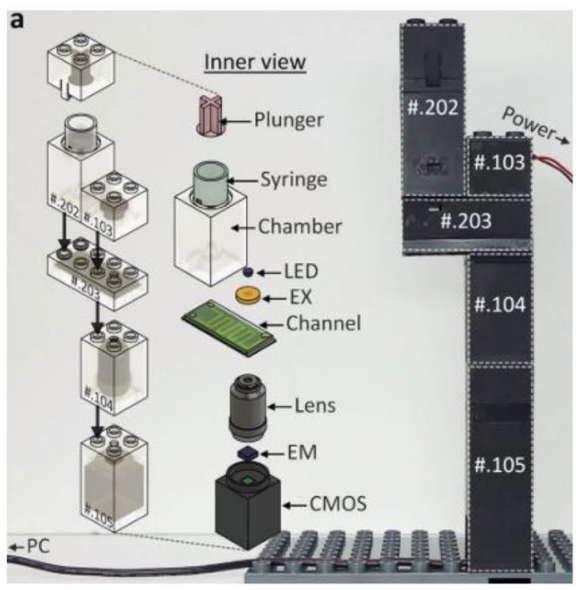

(c)
Fig. 2 a Integrated fluorescence microscope, $2.4 \mathrm{~cm}^{3}$ volume, $1.9 \mathrm{~g}$ weight. b Compact handheld quasi-confocal laser induced fluorescence. c Integration of LEGO-like blocks hosting elements of the optical, electrical, and fluidic circuits in order to modulate the opto-

system was built for high-speed capillary electrophoresis (CE) and it integrated in a modular way a picoliter scale sample injector, short capillary-based fast CE, highvoltage power supply, orthogonal laser induced fluorescence (LIF) detection, battery, system control, on-line data acquisition, processing, storage, and a display. The proposed architecture was embedded into a monolithic black acrylonitrile butadiene styrene (ABS) block of $44 \mathrm{~mm} \times 42 \mathrm{~mm} \times 40 \mathrm{~mm}$ dimension, the overall fabrication cost reaching $\$ 150$. The optical circuitry included a high intensity laser diode (18 mm length, $12 \mathrm{~mm}$ diameter, $80 \mathrm{~mW}$ ) as light source, two small collimating lenses for laser beam focusing and fluorescence collection, and a photodiode ( $9 \mathrm{~mm}$ length $\times 6.2 \mathrm{~mm}$ diameter) as optical detector. The collimating lens consisted of three lenses and had a small size of $8.7 \mathrm{~mm}$ length $\times 9.6 \mathrm{~mm}$ diameter and $7 \mathrm{~mm}$ focal distance. A focusing spot of $10 \mu \mathrm{m}$ for the laser beam could be obtained at the center of a capillary channel. The photodiodes achieved a larger degree of miniaturization compared to the photomultipliers, but the sensitivity was lower.

A design based on a reflective sphere, aiming at enhancing the excitation and collection efficiency, was proposed by Harmon et al. (2018). The interrogation volume was located in the center of a sphere, to produce effects that increased the collection efficiency. A hole was drilled in the sphere, perpendicular to the direction of a microchannel, serving as a light guide from the source located outside of the sphere. The incident light intersected the sample in the region of the interrogation volume, and the reflected light from the walls of the sphere refocused on fluidic systems. (a) Reprinted from (Ghosh et al. 2011), Copyright 2011, with permission from Nature Publishing Group. (b) reprinted from (Fang et al. 2016), Copyright 2016, with permission from Elsevier. (c) Reprinted from (Lee et al. 2018), Copyright 2018, with permission from Willey

the sample, rendering the excitation more efficient. A similar arrangement was available for the fluorescence that was collected through another drilled channel in the sphere.

A cost-efficient device built from off-the-shelf components, including a commercially-available CMOS-based universal serial bus (USB) camera was reported by Zhang et al. (2015). Four poly(methyl methacrylate) (PMMA) sheet frames with holes near the edges, for bolts/screws, were fabricated to serve as a layered modular support for the optical components. Screws were used to adjust the distance between the base and sample holders. The bottom PMMA layer contained the CMOS sensor. The lens was inverted to obtain magnification rather than the de-magnification mechanism of the camera. Resulted modularity allowed the integration with a large variety of preexisting platforms (e.g. cell culture plates, microfluidic devices, and organs-on-a-chip systems).

Time-resolved fluorescence is a methodology implemented for improving the sensitivity of the detection by sequentially powering the emitting source on and off to avoid autofluorescence or excitation light reaching the detector while fluorescence is quantified. Biotechnology, electronics and chemical technology were combined in $\mathrm{Zi}$ et al. (2016) to develop an integration scheme for a time-resolved fluorescence sensing system. Off-the-shelf miniaturized modules (ultraviolet light-emitting diode (UV-LED), silicon photodiode, signal processing units, displays, and optical paths) were integrated into a detection platform of $26 \mathrm{~cm} \times 20 \mathrm{~cm} \times 13 \mathrm{~cm}$. This instrument was used for quantification of analytes in human blood and other body fluids. 
Modular optofluidics is the solution proposed by Brammer and Mappes (2014) and by Lee et al. (2018), among others, aiming to make the widespread implementation of the microfluidic systems more reliable (see Fig. 2c). Lee et al. (2018) proposed a strategy based on modular blocks embedding the elements of the optical, electrical, and fluidic circuits into LEGO-like structures fabricated using rapid prototyping fabrication techniques.

\section{Towards on-chip fluorescence sensing architectures}

On-chip systems are defined as structures that have all the electrical, optical, chemical, and detection functions integrated within the microfluidic chip platform (Kuswandi et al. 2007). This integration strategy that combines optics and microfluidics, resulting in highly efficient liquid-solid interactions created the field of optofluidics (Yang and Gijs 2018). The development process of integrated circuits established technological methodologies that have been successfully used to solve issues in fields outside of electronics, generally known as the MicroElectroMechanical Systems or MEMS industry (Wu and Gu 2011). In this way, routes towards new technological fields appeared, such as micromechanics, microfluidics and micro-optics (integrated optics) (Hierlemann et al. 2003). Soon after this, the limitations of integrated circuits on silicon chips (material mainly used for the development of integrated circuits) became apparent. The requirements of electronics frequently collided with those of optics and mechanics, so that integration on one and the same chip (monolithic integration) proved to be hard (Gründler 2007). Thus, engineers had to consider new integration methodologies and hybrid technologies have been envisaged, i.e. the final device was composed of complex subunits, which were manufactured by different technological processes. Polymer are here considered due to their ease-of-fabrication and cost-efficiency. Within this section, a brief description of the latest achievements towards on-chip integration of fluorescence detection using hybrid technologies is provided.

\subsection{Optical-fiber-based designs}

Implementation of optical fibers based designs in fluorescence sensing (see Table 2) can be observed from two perspectives: (a) full-core optical fibers, and (b) microstructured hollow-core optical fibers. Integration of full-core optical fibers as waveguides intermediating the light transfer from external excitation sources towards the sample volume and/ or the fluorescence transfer towards external photon detector has been one of the initial techniques used in fluorescence sensing miniaturization. Actualized theoretical aspects related to the implementation of optical fibers in fluorescence biosensing applications could be found in a comprehensive review (Benito-Peña et al. 2016).

Optical fibers were adopted as waveguides in this field due to the fact that they are characterized as high light transmission efficient, low-cost, free from electronic noise, providing protection for the electronic components when dangerous chemical environments are present (Yue et al. 2015; Yeh et al. 2017). However, their main drawback results from the fact that they are not user-friendly, requiring precise alignment, with coupling being sensitive to vibrations. Some recent works treated this topic (Matteucci et al. 2015; Rodríguez-Ruiz et al. 2015), proposing some fabrication methodologies allowing free and relatively precise alignment of the fibers. For example, (Matteucci et al. 2015) introduced a fabrication methodology that embedded standard commercially-available optical fibers with Cyclic olefin Copolymer (CoC) TOPAS 5013 hard-polymer chips, based on an injection molding technique (i.e. production-friendly). TOPAS 5013 polymer possesses characteristics that are compatible with the demands of the optofluidic chips. This polymer is highly transparent in the visible spectrum, possessing a high glass transition temperature $\left(140{ }^{\circ} \mathrm{C}\right)$, very low water absorption, is resistant to acids, alkaline agents and polar solvents, and avoids biofouling (minimal surface treatments are needed). The autofluorescence of TOPAS 5013 could be decreased to values that are $20 \%$ lower than the ones resulting in silica material by the addition of a master-batch of a blank dye to the polymer granulate used for injection molding of the devices (Østergaard et al. 2015). Hatch et al. (2014) used optical fibers with ball lenses at their tip, enhancing the light optical density, enabling the implementation of cost-effective and low-power LEDs as light sources. The device was portable, but still bulky relative to the current integration trends.

Even if the current trend in the miniaturization of optical sensing is to bring the light source and the photon detector in the close vicinity of the interrogation sample, standard optical fibers are still used for those cases where robust modular systems are desired. Three-dimensional modular microfluidics has been recently introduced (Bhargava et al. 2014) as a methodology to overcome the issues appearing when monolithic design strategies fail. Modularity is one of the solutions envisaged for bringing microfluidic devices closer to its large-scale commercial implementation. Thus, standard optical fibers are still used nowadays for the development of modular and reliable miniaturized fluorescence-based optical sensors. Ackermann et al. (2016b) introduced the Chip-to-World Interface (CWI) as a plug-and-measure sensing strategy based on a $\mathrm{CO}_{2}$-laser machining fabrication technique, to create a low-cost and robust modular interface facilitating the connection to a large variety of external photon detectors and 


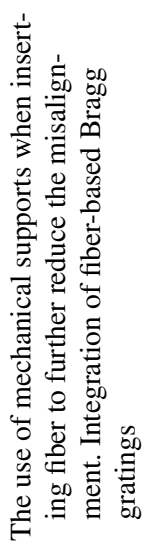

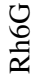
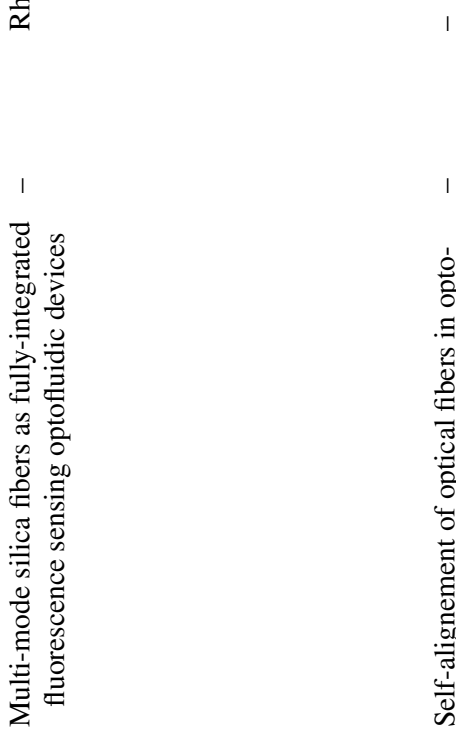

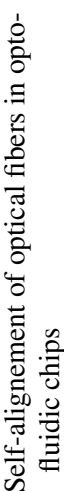

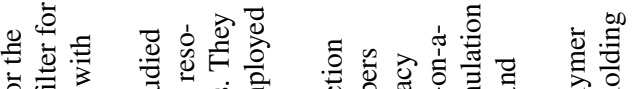

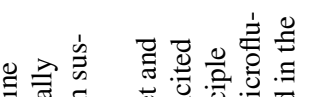

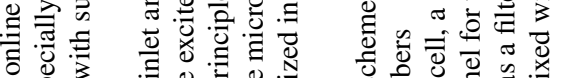
$\circlearrowleft$ 舟

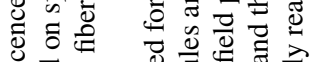

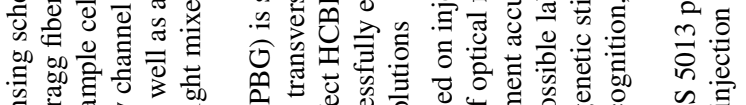

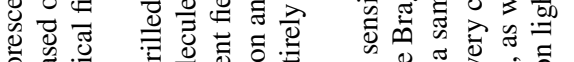

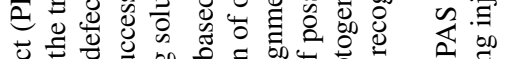

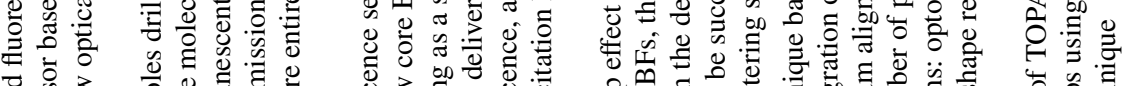

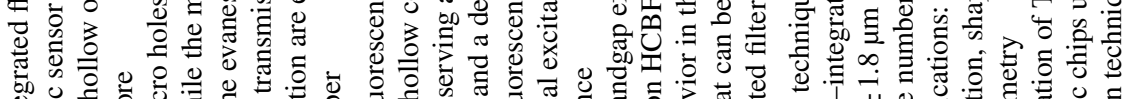

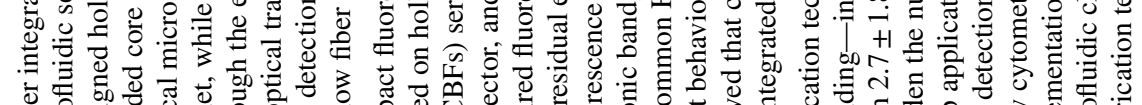

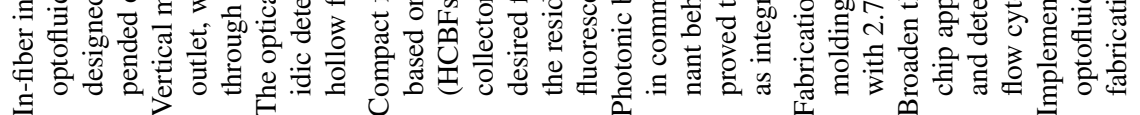

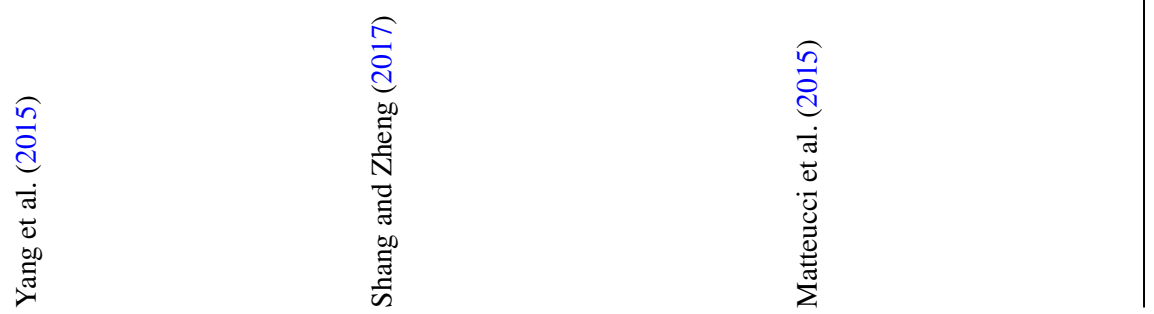




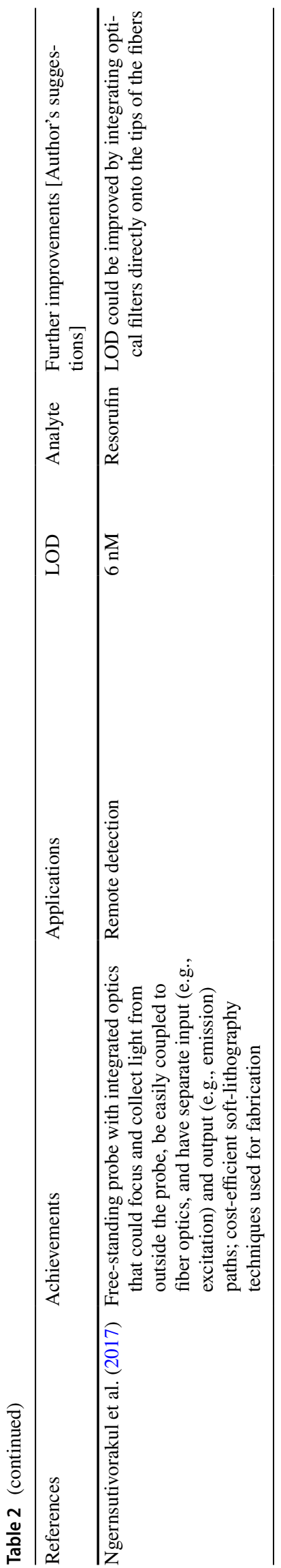

light sources. This concept refers to the implementation of an optical fiber with coaxial radio frequency subminiature version A (SMA) connectors, allowing manipulation of the detection system without special training of a potential user. Yue et al. (2015) used also optical fibers with SMA connectors to develop a modular fluorometer for detection of fluorescein isothiocyanate. A concentration of $10 \mathrm{ng} /$ $\mathrm{mL}$ could be measured, and the system presented a good linearity from $10 \mathrm{ng} / \mathrm{mL}$ to $10 \mu \mathrm{g} / \mathrm{mL}$. The opto-electrical converter module and the signal acquisition device were modular and could be replaced for the detection of other molecules. Moreover, an optical probe is a tool used to collect light from otherwise inaccessible volumes. Ngernsutivorakul et al. (2017) proposed a $0.5 \mathrm{~mm}$ thick and $1 \mathrm{~mm}$ wide PDMS probe with two optical fibers integrated as inlet and outlet light waveguides. In addition, the probe embedded pre-aligned mirrors, lenses, allowing separate input and output optical paths. The probe was disposable, reusable from one chip to another, but not alignment-free, requiring a specific instrument to couple it to a microfluidic chip.

Next implementation level of optical fibers in the miniaturization process of fluorescence sensing is represented by the microstructured optical fiber (MOF) or photonic crystal fiber (PCF). The field of optofluidics in microstructured optical fibers was recently reviewed in Shao (2018) and Ertman et al. (2017). It gained high interest due to its capability of simplifying optical fiber sensors and improving the level of integration. Hollow-core Bragg fibers (HCBFs) working on the principle of evanescent wave are a promising solution that might converge towards a fully integrated detector $(\mathrm{Li}$ and Nallappan 2019). HCBFs form a particular class of photonic bandgap (PBG) fibers (Huang et al. 2004) with a guiding mechanism that is capable of confining the light in the fiber core from all incidence angles and polarizations. Due to the PBG effect, the HCBFs have features of a wideband band-pass filter. The Bragg layers can be designed in such a way that they behave as a reflective surface for specific wavelengths and absorbing surface for others. By introducing a defect layer into the common cladding band, the excitation light is rejected, while fluorescence light is transmitted towards the photon-sensing element. Also, the rejection filtering is narrowed due to the transverse resonant behavior, explained elsewhere (Chen et al. 2008). Shang and Zheng (2017) applied this principle, resulting in a very compact detection device where the detection cuvette, the collector, the delivery channel for the desired fluorescence, and the filter for residual excitation light mixed with fluorescence were all part of the fiber (see Fig. 3). In Yang et al. (2015), a hollow optical fiber with suspended core was proposed, fabricated, and characterized for detection of Rhodamine $6 \mathrm{G}$. The outer diameter of the fiber was $350 \mu \mathrm{m}$, the inner diameter of the fiber was $210 \mu \mathrm{m}$ and the core diameter was 
(a)

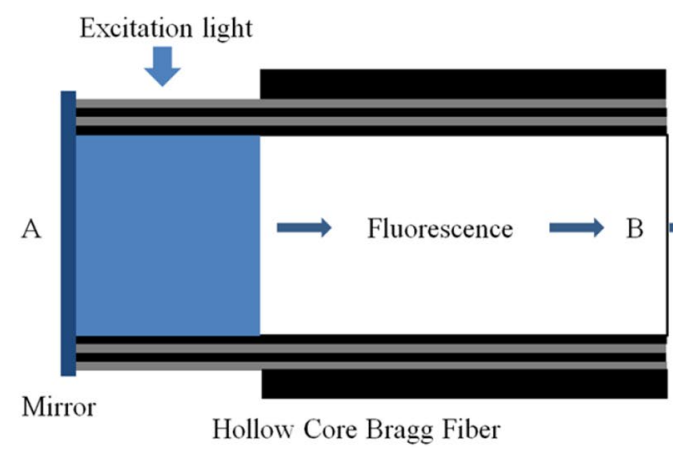

(b)

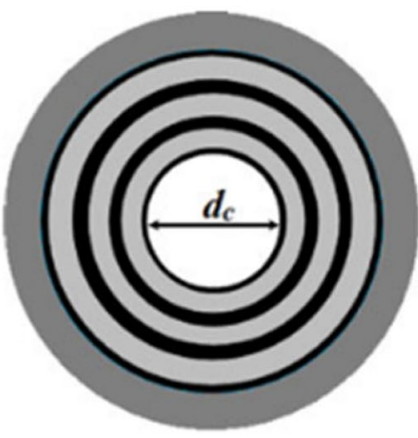

Fig. 3 a Compact fluorescence-sensing scheme based on hollow core Bragg fibers, serving as interrogation cuvette, collector, delivery channel for the fluorescence, and the filter for the residual noise. $\mathbf{b}$

$40 \mu \mathrm{m}$. The inlets and outlets were made on the surface of the hollow fiber by $\mathrm{CO}_{2}$ laser etching, all the others optical elements being off-chip.

\subsection{Optics layer-integrated designs}

The basic principle of this strategy is to integrate the active and passive optical components on a planar layer, mainly by implementing the micro- and nano-fabrication technologies (Dandin et al. 2007). Transparent materials with high refractive indexes, such as polymers, are usually used as waveguides and the light remains confined by multiple total internal reflections (Gründler 2007). Some of the main active current research directions are in-plane microfluidic lenses, printed-board microfluidics, antiresonant reflecting optical waveguides (ARROWs), and on-chip integration of solid-state light emitters (SSLE). In-plane microfluidic lenses or on-chip lens systems mainly aim to robustly integrate light beam focusing customized solutions in the same functional layer as the fluidic layer (Bates and $\mathrm{Lu} 2016$ ). Liquid-core cladding lenses, pressure-controlled liquid-air interface and gradient refractive index lenses are some configurations recently reported in the literature tackling this subject (see Table 3).

Femtosecond lasers revolutionized the three-dimensional micro-fabrication of the materials due to their very short pulse width and high peak intensity. Known as a complicated technique, recent advancements proved the femtosecond laser micromachining as a cost-effective and reliable fabrication technique for optofluidic systems (Sugioka and Cheng 2012; He et al. 2014; Sugioka et al. 2014; Gu et al. 2015; Joseph et al. 2017; Serhatlioglu et al. 2017). An inplane integrated microfluidic lens whose modulation could be on/off switched on demand was fabricated by Paiè et al. (2017) using femtosecond laser micromachining (FLM) fabrication technique. Modulation of the light is a common technique used to enhance the measurement sensitivity by
Cross-section scheme of the device with the photonic band gaps of different refraction indexes (Shang and Zheng 2017)

subtracting the noise. The focused light could be modulated by dynamically changing the liquid in the lens through a droplet generation module. Femtosecond laser micromachining and radio frequency (RF) sputtering were implemented for the incorporation of a microfluidic network, excitation, filtering, and collection elements in one glass substrate, in a $90^{\circ}$ configuration (Guduru et al. 2016). Here, the FLM was used to fabricate the microfluidic channel, the perpendicular fiber channels, perfectly aligned and embedded in the fused silica layer. A wavelength filter, behaving as a Bragg mirror, was fabricated by implementing an RF sputtering method. One-dimensional photonic crystals were used, since they are dielectric structures of different refractive indexes, permitting propagation of only specific wavelengths. They can be designed to have a photonic bandgap in the wavelength region of excitation, similar to the concept explained in the previous subchapter. On the top of the filter, a binary Fresnel lens (BFL) was fabricated, leading the collected light out of the chip. Fresnel lenses are flat, low thickness structures with concentric rings designed to focus the light (40\% efficiency) in optical microfluidic devices (see Fig. 8b) Their fabrication within a microfluidic channel is based on the nanoimprint process (Siudzinska et al. 2017). Efficient beaming of the emitted radiation is very important since it can drastically affect the performance of the assay. Ricciardi et al. (2015b) proposed an optofluidic chip based on a multilayered photonic crystal structure embedded on a PDMS structure. The efficiency of fluorescence collection was experimentally demonstrated in an antibody-antigene immunoassay, resulting in a decrease of the limit-of-detection (LOD) for labelled antigenes by a factor of about 40 . The measurements were demonstrated to occur in a robust and reproducible way, with reduced optical alignment issues. Here, it deserves mentioning the method proposed by Watts et al. (2012) for an efficient beaming of the excitation light. This method eliminated the need for free-space optics and high-quality light sources, through on-chip 3D hydrodynamic focusing. 


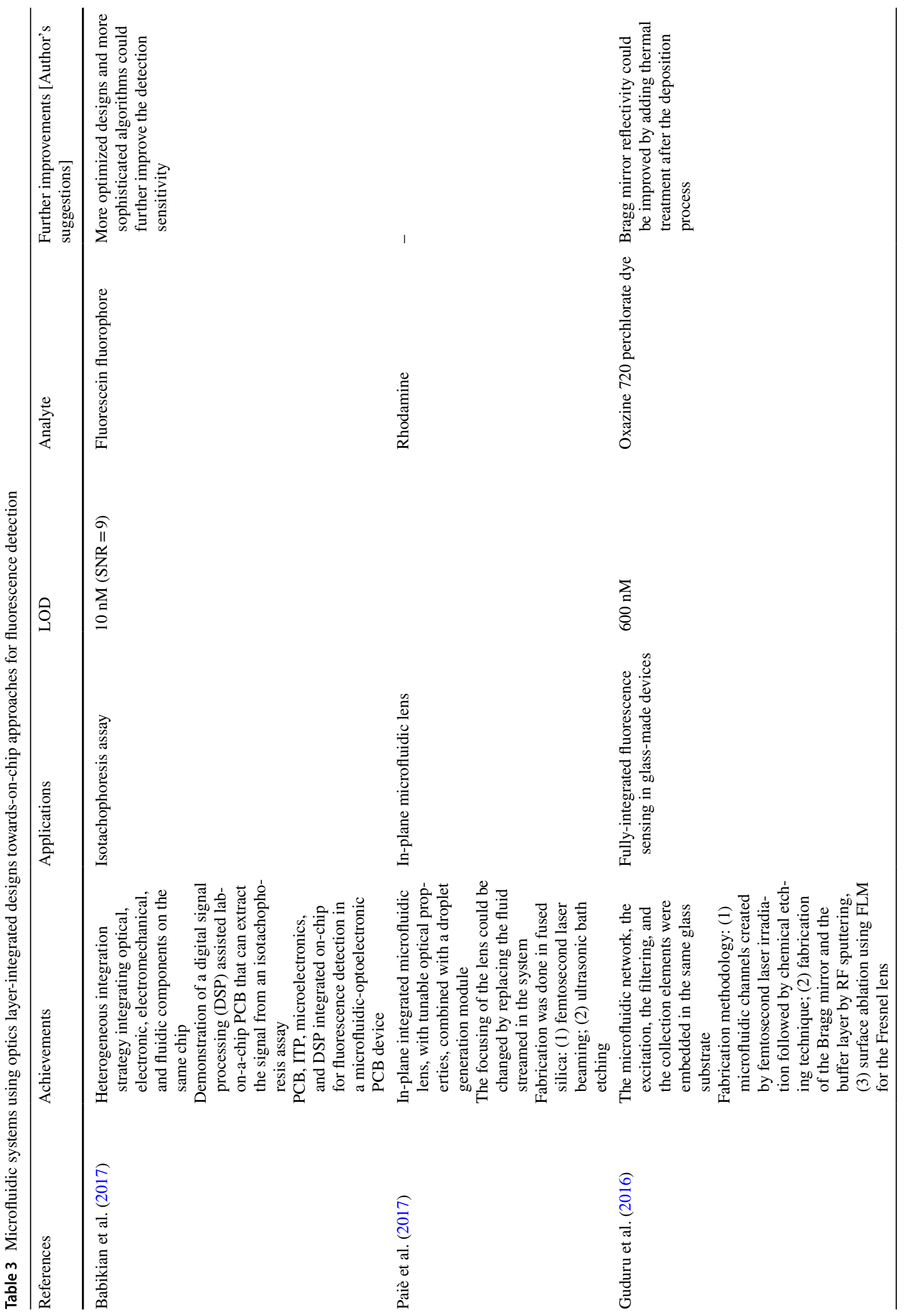



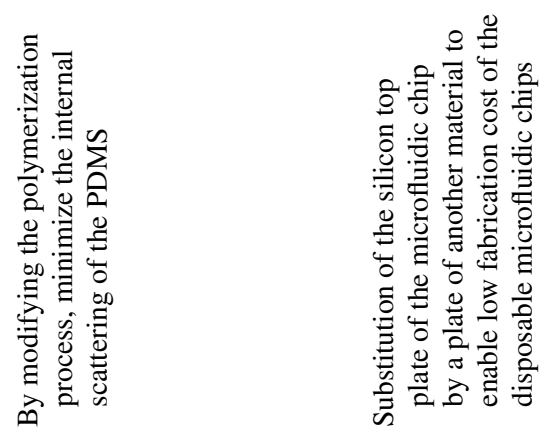

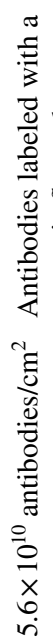

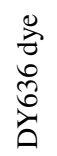

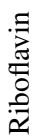

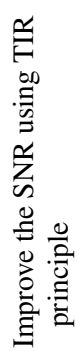

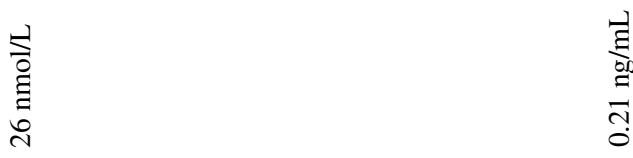

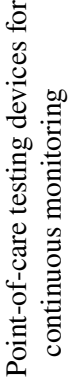

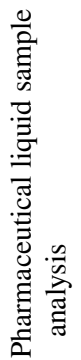

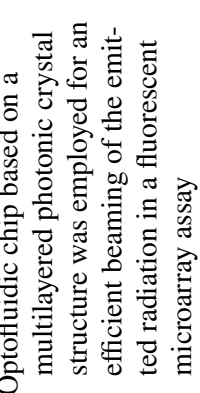

- च छ

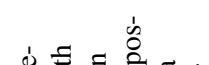

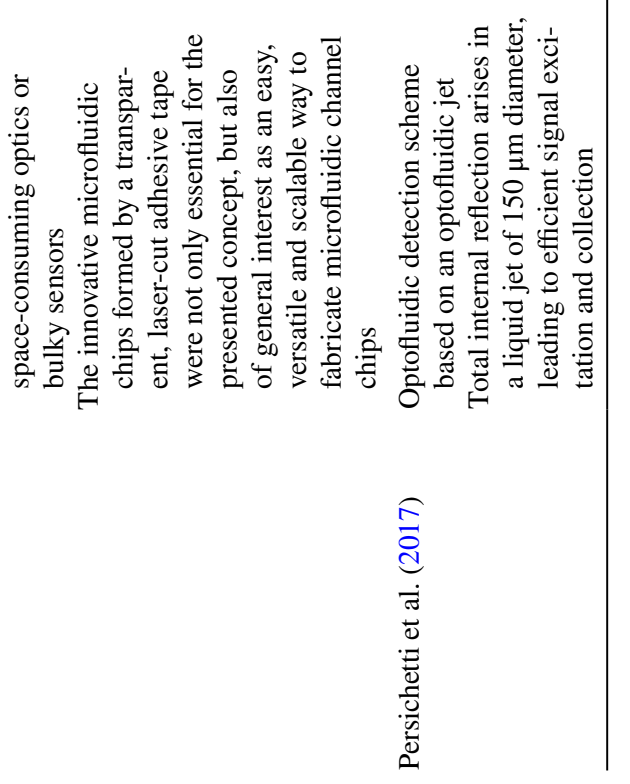




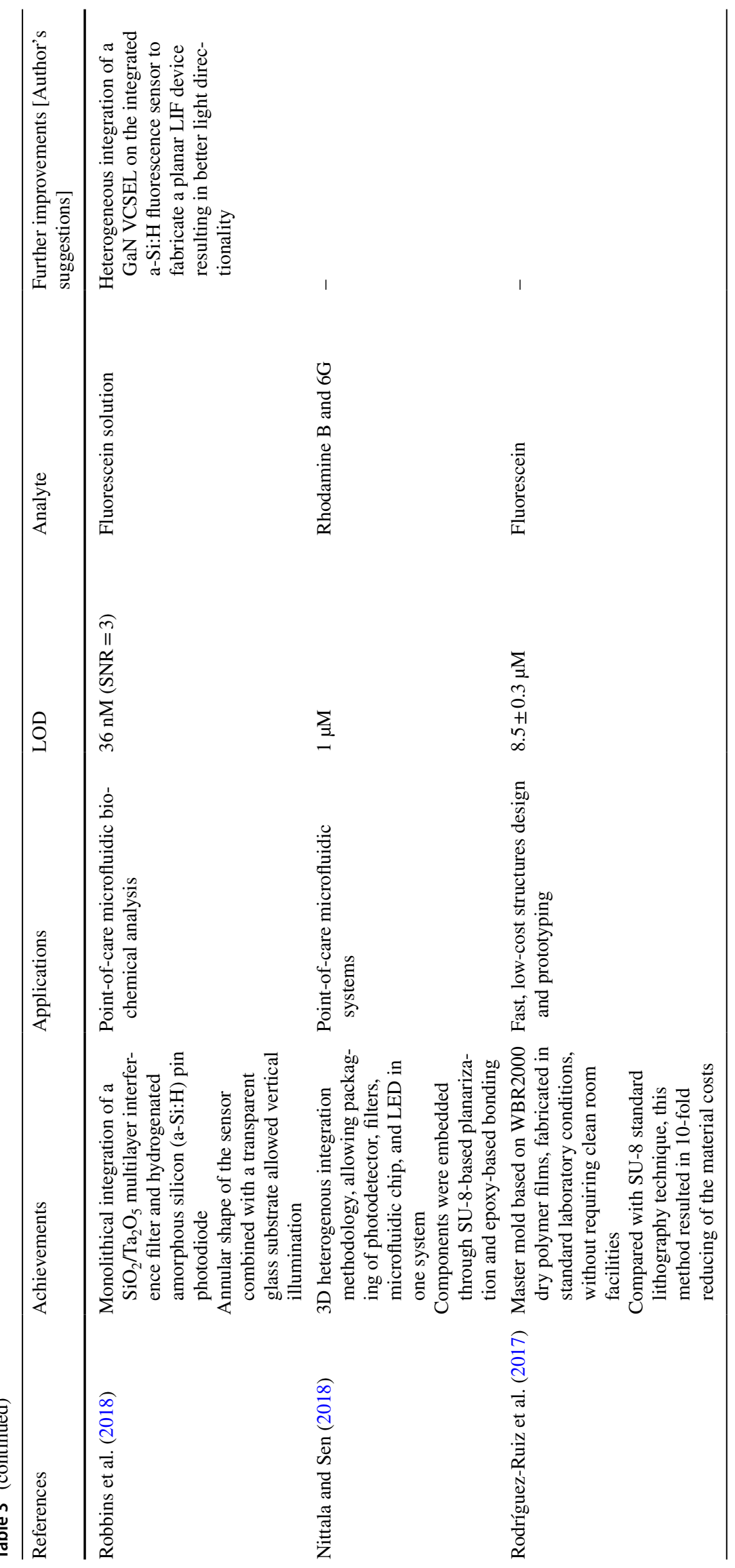


The on-chip hydrodynamic focusing is achieved through the introduction of micro-patterned optical components in an epoxy-based SU-8 photoresist layer that hosts also the fluidic components.

The printed circuit board (PCB) is a mature technology used and improved for decades in electronics, to mechanically support and electrically connect components using conductive tracks etched in layers of copper onto and/or embedded between sheets of a non-conductive substrate. The materials usually used as substrates for the fabrication of PCBs are resistant at high temperatures $\left(>170{ }^{\circ} \mathrm{C}\right)$, this characteristic making them compatible with microfabrication techniques that involves elevated temperatures. The reliability and potential of PCBs for miniaturization of fluorescence sensing systems was demonstrated recently (Novo et al. 2014; Shin et al. 2015, 2017; Babikian et al. 2017; Obahiagbon et al. 2018). A PCB offers an integration alternative to glass/polymeric microfluidic chips, which are not integration-friendly with standard, off-the-shelf optoelectronic elements (Babikian et al. 2017).

Portable systems integrating light-emitting diodes (LEDs) for multiple target analysis were proposed in Shin et al. (2015, 2017). Optical functions were modularly parallelized within three layers (see Fig. 4a, c). The first layer (filtering and detection layer) hosted a photon detector, a dichroic lens, and a color filter. The intermediate layer was represented by a PCB with three LEDs of different wavelengths mechanically mounted around a circular orifice, which illuminated the interrogation volume from the opposite direction of the photon detector. Avoiding direct illumination of the detector was relevant for increasing the signal-to-noise ratio. The circular cut assured that the emitted fluorescence was transferred from the third layer (interrogation volume) to the first layer (filtering and detection layer), while the PDMS microfluidic layer was disposable. All above-mentioned components were embedded into a portable box equipped with a display, permitting the selection of the operation. A comprehensive review about LEDs implementation with fluorescence sensors is reported in Yeh et al. (2017).

As a predictable continuation of the above-mentioned concepts, the microfluidic-PCB concept was introduced by Babikian et al. (2017). This concept involved a three-layer device, integrating with the aid of two PCB parts, all the fluidic, electrical, mechanical and optical components within a compact system (see Fig. 4b). One PCB was fixed and non-disposable, comprising all active components, and was denoted the reader chip. The second PCB, named the microfluidic chip, was disposable and encompassed the passive structures of the device such as microchannels, high voltage electrodes, and micromachined light reflector. A standard surface-mounted blue LED was used as a light source. The detection was implemented using a standard surfacemount 2-megapixel CMOS imaging array with integrated red-green-blue (RGB) filters. The CMOS sensors were inexpensive, compact, and compatible with the microfluidicPCB approach. A surface-mounted light reflector was used to reflect the excitation light path towards the detection channel. A rectangular slit was created in the upper PCB, above and along the microfluidic channel, to avoid light scattering and autofluorescence of the PCB substrate. The background noise associated with the excitation light was further suppressed by a Söller collimator film, laminated on top of the CMOS pixel array. A Söller collimator collimates light by allowing the passage of rays that are almost parallel with its optical axis and it is used in lens-free fluorescence detection schemes since uniform spatial distribution of light was

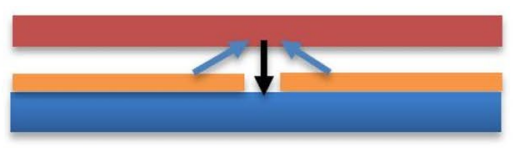

Microfluidic layer

Printed Circuit Board

Filtering and detection layer

(a)

$\rightarrow$ Excitation beam from LED

Fluorescence beam from detection chamber

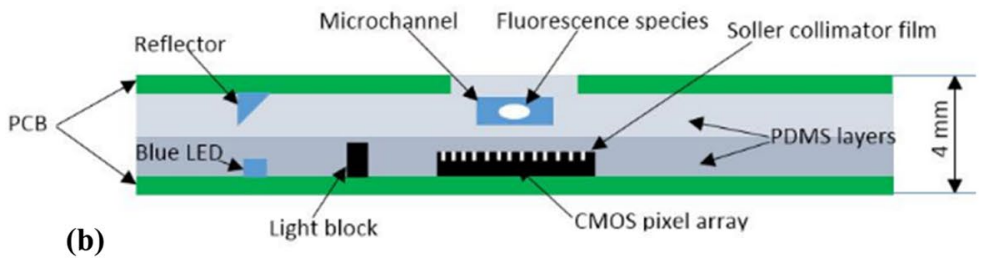

(c)

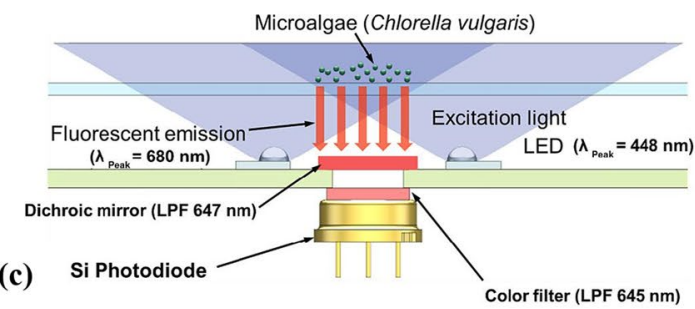

Fig. 4 a Parallel layering distribution of the detection functions with the PCB sandwiched in between the microfluidic layer and the detection layer (Shin et al. 2015, 2017). b Cross-section of the microfluidic-PCB fluorescence detector, built using two PCBs. The design strategy avoided the direct illumination of the interrogation volume, a Söller thin film collimator being coated on top of the CMOS to reduce the background noise (Babikian et al. 2017). c Fluorescence detection system with backside illumination scheme. Reproduced from (Shin et al. 2015, 2017), Copyright 2015, with permission from Elsevier 
crucial (Balsam et al. 2012). In this work, it also avoided the cross-talking between the light source and the CMOS image sensor. This heterogeneous method embeds individually manufactured components, such as electrodes, heaters, light sources, detectors, and microfluidic components between the electrical and microfluidic layers using standard fabrication processes. All the components (light source, microchannel, optical guide, and detection sensor) were integrated within a structure having the approximate dimensions of a credit card, $7 \mathrm{~cm} \times 5 \mathrm{~cm} \times 4 \mathrm{~mm}$.

Total internal reflection (TIR) takes place when a light beam meets a medium with a lower refraction index at an angle greater than the critical angle. This critical angle can be calculated knowing the refraction indexes of both media. The phenomenon can be used to mitigate direct illumination of the detector and, by consequence, to lower the noise level. For example, a design solution using a lateral excitation configuration, to (1) maximize the photon flux exciting the microfluidic channel while (2) preventing excitation light reaching the sensor, was proposed by Novo et al. (2014). This configuration (see Fig. 5a) involved a prism-like polydimethylsiloxane (PDMS) microchannel sealed with a glass substrate. A printed circuit board hosted a micro-fabricated a-Si-H photodiode and contained a two-level pocket, which kept a $50 \mu \mathrm{m}$ space between the microfluidic chip and photodiode needed for the total internal reflection condition. A
$405 \mathrm{~nm}$ laser beam was directed perpendicular to the lateral prism-like PDMS structure in which the sides made a $70^{\circ}$ angle with the flat surface and was focused to illuminate the microchannel while experiencing total internal reflection at the glass-air interface. This configuration improved the detectability range by two orders of magnitude as compared to a normally incident excitation configuration, signal-to-noise ratio being ameliorated for detection of specific fluorophores.

Berner et al. (2017) developed a method based on sandwiching laser-cut double-sided adhesive tapes coupled with the latest generation of thin-film photodetectors, enabling miniaturization by the custom fitting of amorphous siliconbased photodiode arrays to the geometry of the flow channel.

One promising methodology using TIR phenomena was presented in Jang and Yoo (2013), where a fluorometer integrating a total internal reflector was introduced, a condensing mirror and the detection chamber (width $1.5 \mathrm{~mm} \times$ depth $0.8 \mathrm{~mm} \times$ length $5 \mathrm{~mm}$ ) within a single $1.2 \mathrm{~mm}$ thick polycarbonate substrate. The total internal reflector enabled orthogonal detection, and the condensing mirror increased the selectivity of the fluorescence emission. The limit of detection achieved was $5 \mathrm{nmol} / \mathrm{L}$ and the linearity was 0.994 for 6-FAM fluorescence dye. The condensing mirror was coated with a $200 \mathrm{~nm}$ thick aluminum layer, having the role of better fluorescence collection from the sample and more
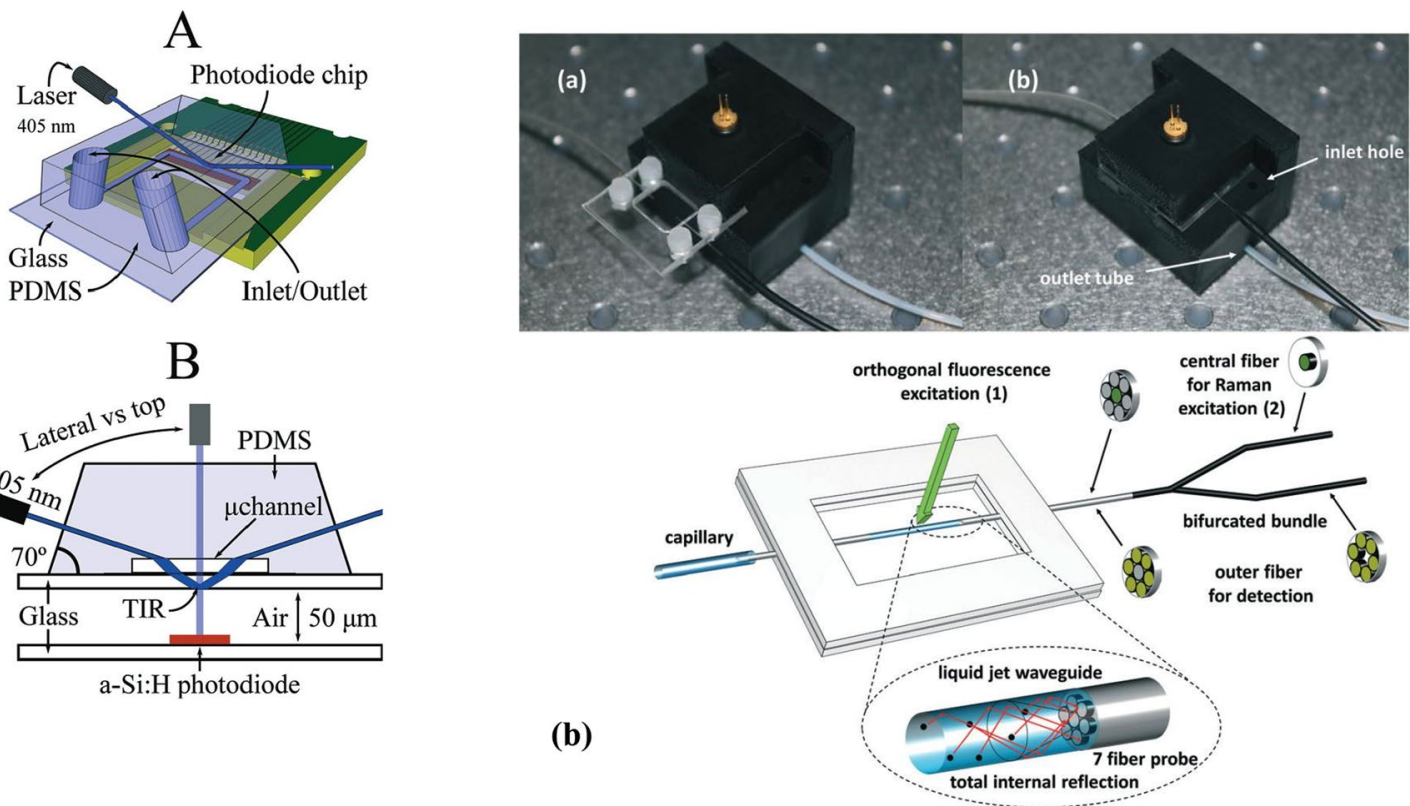

Fig. 5 a Lateral excitation onto a PDMS microfluidic device coupled to a photodiode PCB. The $50 \mu \mathrm{m}$ air-gap separates the microfluidic device from the photodiode, in order to assure the TIR effect at the glass/air interface for the excitation light. b Multi-functional sensing device based on $150 \mu \mathrm{m}$ optofluidic jet, equipped with a recirculation system. The detection scheme is based on the principle of the TIR in

the liquid jet. Two possible ways of liquid jet excitation: (1) orthogonal fluorescence excitation, and (2) optical fiber for Raman excitation. (a) Reproduced from (Novo et al. 2014), Copyright 2014, with permission from The Royal Society of Chemistry. (b) Reproduced from (Persichetti et al. 2017), Copyright 2017, with permission from The Royal Society of Chemistry 
efficient distribution to the photon detector, located on the opposite side of the fluidic chamber, while the light source (465 nm LED) and the photon detector were externally located. A system based on an optofluidic jet waveguide (see Fig. 5b) — a liquid micro-jet with $150 \mu \mathrm{m}$ diameter-leading to highly efficient signal excitation and collection was introduced by Persichetti et al. (2017).

Alignment of the light emitter and the optical circuit is a very sensitive part of an optofluidic system. Small variations (less than 5\%) may produce large performance variations that make the system unreliable (Llobera et al. 2015). Thus, monolithic integration of the solid-state light emitters (SSLEs) is a field of high importance. Llobera et al. (2015) proposed an innovative hybrid monolithic solution (see Fig. 6a) for on-chip integration of a SSLE aligned with a multiple internal reflection (MIR) system. The SSLE was made of a fluorophore-doped hybrid xerogel material. The fabrication procedure involved a low-cost photolithographic fabrication step. Air mirrors were made to assure light coupling from the light source to the MIR system. Robbins et al. (2018) introduced a compact device embedding a $\mathrm{SiO}_{2} /$ $\mathrm{Ta}_{2} \mathrm{O}_{5}$ multilayer optical interference filter, a hydrogenated amorphous silicon (a-Si:H) pin photodiode, an asymmetric microlens, and a GaN micro LED. The system was capable to collect fluorescence light in a $100 \mu \mathrm{m}$ microfluidic channel, the device reaching a $36 \mathrm{nM}$ limit of detection for fluorescein solution, but the integration of the micro LED was concluded as being difficult due to its lack of directionality. However, the fabrication procedure paved the way towards planar heterogeneous integration of GaN micro LED on an a-Si:H fluorescence sensor. Kang et al. (2016) developed a fabrication methodology (see Fig. 6b) for building mechanically flexible microfluidic fluorescence sensors. They managed to integrate microscale vertical cavity surface-emitting lasers (micro VCSELs) and silicon photodiodes on a flexible substrate of polyethylene terephthalate (PET). This substrate integrated with elastomeric fluidic chips on plastics demonstrated potential for multiplexed, real-time operation.

Polymers or silicon-based materials with refractive indexes varying between 1.4 and 3.5 are usually used for the fabrication of optofluidic integrated systems. Since the refractive index of water is 1.33 , the total internal reflection condition required to confine the light when hollowcore optical waveguides are used simply cannot be satisfied for this particular case. Antiresonant reflecting optical waveguides (ARROWs), based on the thin-film interference principle and the conventional silicon microfabrication techniques, propose a solution for this inconvenient. A particular solution is represented by the aerogel waveguides. A series of alternating dielectric layers creates the conditions for interference-based guidance of leaky modes (Parks et al. 2014). This technique proved to be very suitable for very sensitive fluorescence detection. Parks et al. (2014) used this technology for developing a programmable microfluidic chip with on-chip detection of fluorescence. The active control of the biologic solutions was realized using programmable microvalve arrays (see Figs. 6a, 7). Measor et al. (2011) introduced a methodology for the fabrication of on-chip (a)

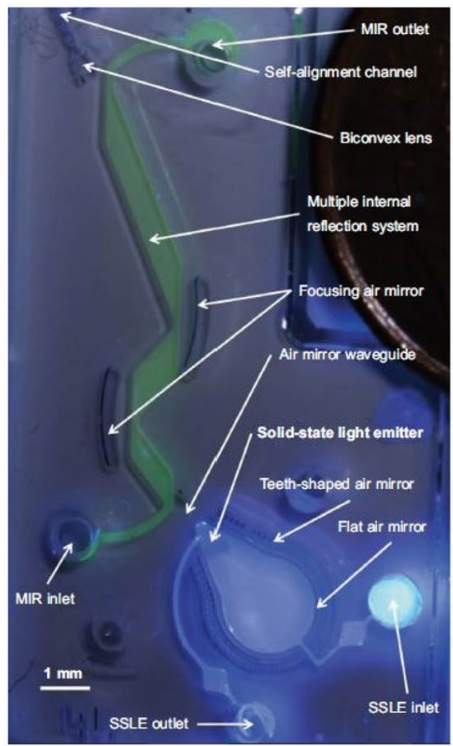

(a)

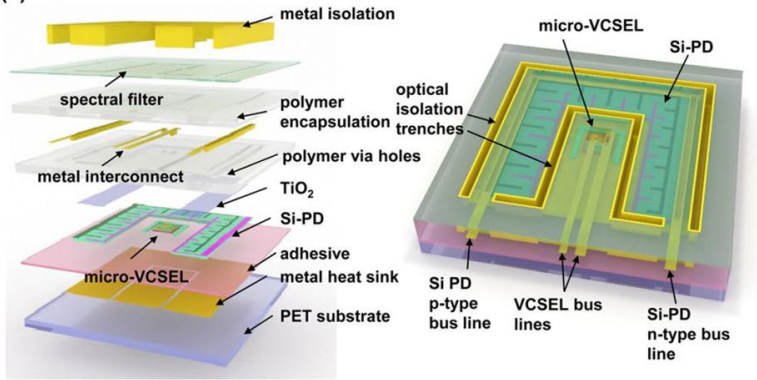

(b)

(b)

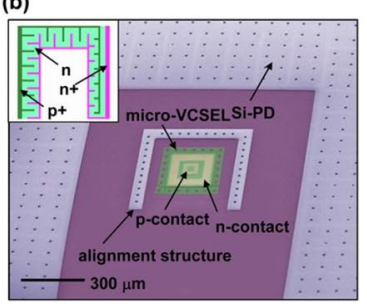

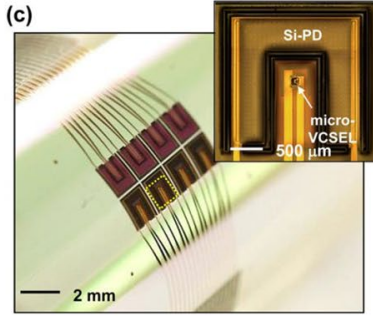

Fig. 6 a Photonic lab-on-a-chip with the solid-state light emitter, air mirrors, multiple internal refection system, biconvex lens, and the channel for fiber optics integration. (b) (a) Illustration of the flexible sensor design and fabrication methodology. (b) Scanning electron microscopy view of the VCSEL and the $3 \mu \mathrm{m}$ thick Si-Photodiode. (c) Image of an array of sensors wrapped around a cylindrical bar. (a) Reproduced from (Llobera et al. 2015), Copyright 2015, with permission from Springer Nature. (b) Reprinted with permission from (Kang et al. 2016), Copyright 2016, American Chemical Society 


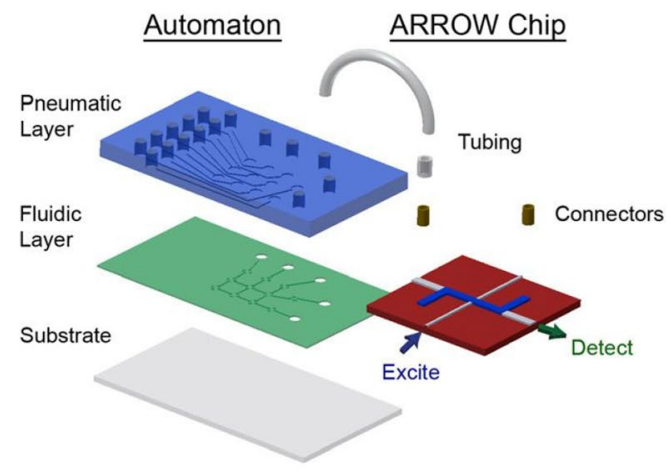

(a)

(a)

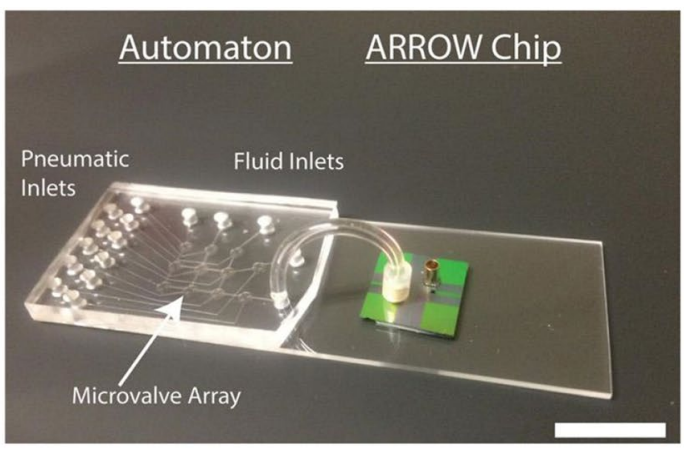

(b)

(b)

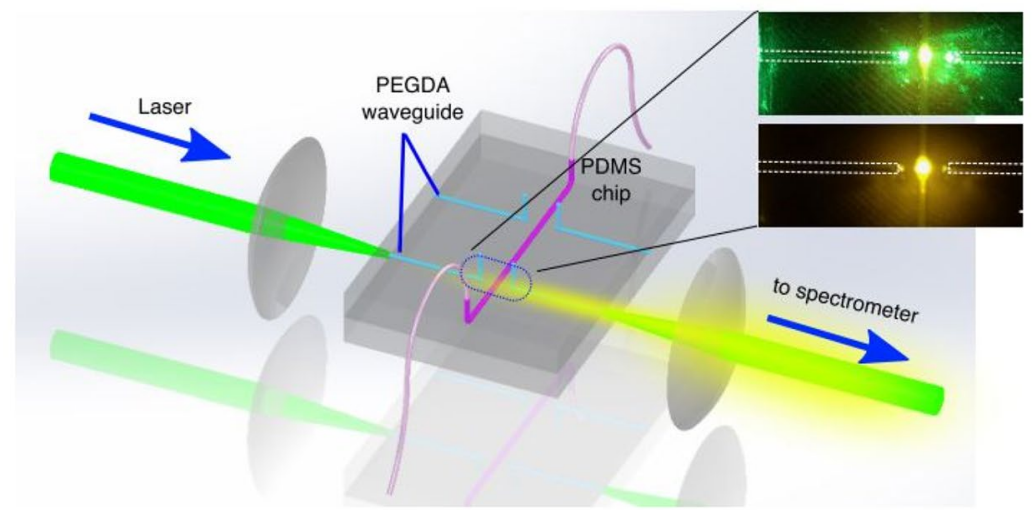

Fig. 7 a Hybrid chip-scale system combining the automated microfluidic processing (automaton) and on-chip optical detection based on the ARROW principle. Scale in the figure $-1 \mathrm{~cm}$. b Optical device schematic with a PEGDA waveguide used for excitation light coming from a $532 \mathrm{~nm}$ laser and another PEGDA waveguide used to collect the fluorescence emission from the interrogation volume. (a) Reproduced from (Parks et al. 2014), Copyright 2014, with permission from AIP Publishing. (b) CC-BY 4.0 interference filters with multiple high and low loss regions. Interference filters, compatible with the silicon microfabrication techniques, possess lower autofluorescence when compared with absorbance filters. In this work, they managed to decrease the number of dielectric layers requested for efficient working of the interference filter from more than 30 down to 3 . Aerogels are materials with low-refractive indexes, making them suitable for solid-cladding in liquidcore optofluidic waveguides based on the principle of total internal reflection. A comprehensive review of aerogels used for optofluidic waveguides is presented in Özbakır et al. (2017).

Following the trend of scaling down by 3D integration of various heterogeneous components, a SU-8 polymerbased methodology was developed by Nittala and Sen (2018). The cost-effective reported methodology allowed integration of commercially available components in a relatively simple way. The key element of this approach was planarization of the layers with correctly chosen SU-8 parameters in such a way that the next layer could be stacked using epoxy-based bonding. This method allowed packaging a photon detector, the filters, the microfluidic chip, and a LED. On the other hand, a low-cost master mold fabrication process based on a dry film photoresist for soft lithography, and operated in standard laboratory conditions was reported in Rodríguez-Ruiz et al. (2017). This protocol managed to reduce by ten times the material costs and reduced considerably the fabrication time compared with standardized SU-8 master mold techniques. The methodology initialized a simpler fabrication methodology of photonic devices. Integrated hydrogel waveguides potential for developing wearable and implantable lab-ona-chip devices was evaluated by Torres-mapa et al. (2019). They used self-aligned polyethylene glycol diacrylate (PEGDA) waveguides, which present tunable mechanical and optical properties, integrated via micro-molding technique into a PDMS structure to evaluate the fluorescence response from a rhodamine $6 \mathrm{G}$ solution. The PEGDA waveguides integrated into the PDMS showed high transmission, minimal absorption in the visible spectrum, and propagations losses lower than $1.1 \mathrm{~dB} / \mathrm{cm}$. Further investigations on mechanical and light guiding properties during stretching/bending are requested (see Fig. 7b). 


\subsection{CMOS-based fluorescence sensing}

CMOS technology revolutionized the field of micro-electronics (Shakoor et al. 2018) and it holds the promise to do so for micro-optical sensing as well. On-chip CMOSintegration, coupled to microfluidics for detection at the micro-scale, was increasingly observed in the recent literature (Nakazawa et al. 2011; Choi et al. 2016; Tanaka et al. 2017; Wei et al. 2017), especially for the development of filterless prototypes (see Table 4). The focusing and the filtration optics represent a barrier in the miniaturization process (Papageorgiou et al. 2017), alternative techniques replacing them being envisaged. The filterless discrimination between the excitation light and the generated fluorescence light emerged as a cost-effective, compact, and lightweight miniaturization method. CMOS technology introduced advantages such as reduced dimensions, high sensitivity, coupled with filtration algorithms, very low unit prices, low-power consumption, and integrated signal processing, making it compatible with the targeted goals of fluorescence detection device miniaturization. Until recently, charged-coupled devices (CCDs) were largely used in image sensing, but now CMOS image sensors seem to be more used, due to their superior features. A CMOS image sensor is an integrated circuit with an array of pixel sensors. Each pixel sensor contains its own light sensor, an amplifier, and a pixel select switch. The main components of a CMOS sensor are color filters, a pixel array, a digital controller and an analog-todigital converter (see Fig. 8a).

Different models are available for gathering information from pixels: RGB; hue, saturation, value (HSV); hue, intensity, saturation (HIS); CIELab or CIExyY (Sudhakaran 2020), all implemented at the system level. There is an increased interest in employing the RGB model combined with an image sensor or a camera to determine the concentration of analytes (Bueno Hernández et al. 2017). The commercially available CMOS image sensors are usually equipped with integrated filters. Generally, the performance of these filters is not sufficient and different filtration algorithms are implemented, to eliminate parasitic light. CMOS-based contact sensing coupled with time-correlated photon counting (TCSPC) has proven to be a sound methodology (Wei et al. 2017). Different strategies implementing CMOS image sensors have been recently proposed Măriuța et al. (2019) with good results and enough space for further improvements. The challenges existing in combining the integrated circuits with biological or chemical components in lab-on-a-CMOS concept, such as thermal effects, floorplanning, signal coupling, electrochemical effects, surface treatments, sterilization, and microfluidic integration were detailed by Datta-Chaudhuri et al. (2016).

One of the most critical challenges in lab-on-a-CMOS design and fabrication represents the interaction between the fluid samples and the chip surface, traditional wire-bonding packaging being not compatible with the planar microfluidic concept. Lindsay et al. (2018) developed a heterogeneous integration solution of a CMOS sensor and a fluidic network using wafer-level molding process. A technology enabling monolithic integration of the read-out system within the sensor for general label-free miniaturized optical detection by integrating nanophotonic structures with CMOS photodiodes was reported in Shakoor et al. (2018). One-dimension grating structures with a CMOS integrated image sensor arrayed with photodiodes. The gratings were made of silicon nitride and refractive index changes were induced when different analytes were applied. Pang et al. (2011) developed a CMOS based detection system by integrating Fresnel zone lenses (above described) (see Fig. 8b).

Tanaka et al. (2017) proposed a system based on charge accumulation techniques that simultaneously allowed filterless measurement of multiple low-intensity fluorescence wavelengths (five in this work). The charge accumulation technique involves fluorescence detection by measuring the voltage change of a capacitor, which is proportional to the accumulated signal charge quantity. This technique has the capability to increase the output signal level, reducing the noise induced by the incident light. From experiments, the dynamic range obtained was 100:1. The filter was fabricated by an older 2-poly, triple-well, $5 \mu \mathrm{m}$ CMOS process.

A lens-free system for breast cancer cell detection was developed by Papageorgiou et al. (2017). It integrated stacked CMOS metal layers above each photodiode to form angle-selective gratings, rejecting background light. Choi et al. (2016) presented a filterless method for the suppression of forward scattering in silicon by surface planarization, resulting in a separation efficiency improving from 550:1 to 1250:1. This was achieved mainly due to the low roughness of the polysilicon surface.

Plasmon enhanced fluorescence or metal enhanced fluorescence, is a powerful amplification method used to increase the sensitivity and shorten detection times, and is based on the interaction between fluorophore labels that are coupled with the confined field of surface plasmons. A comprehensive review of this topic can be obtained from Bauch et al. (2014) and a detailed overview is presented in the book of Geddes (2017). The integration of nano-plasmonics with CMOS technology, without any other post-processing, enabled the possibility to integrate large multiplexed assays on the same chip. A detailed theoretical description of plasmonenhanced fluorescence coupled with CMOS technology is found in Hong et al. (2017a).

3D copper-based nanoplasmonics components were integrated within standard CMOS devices through sub-wavelength copper-based electrical interconnect lithography features by Hong et al. (2017a), developing a bio-sensor with a nano-waveguide array-based filter. The sensor was 


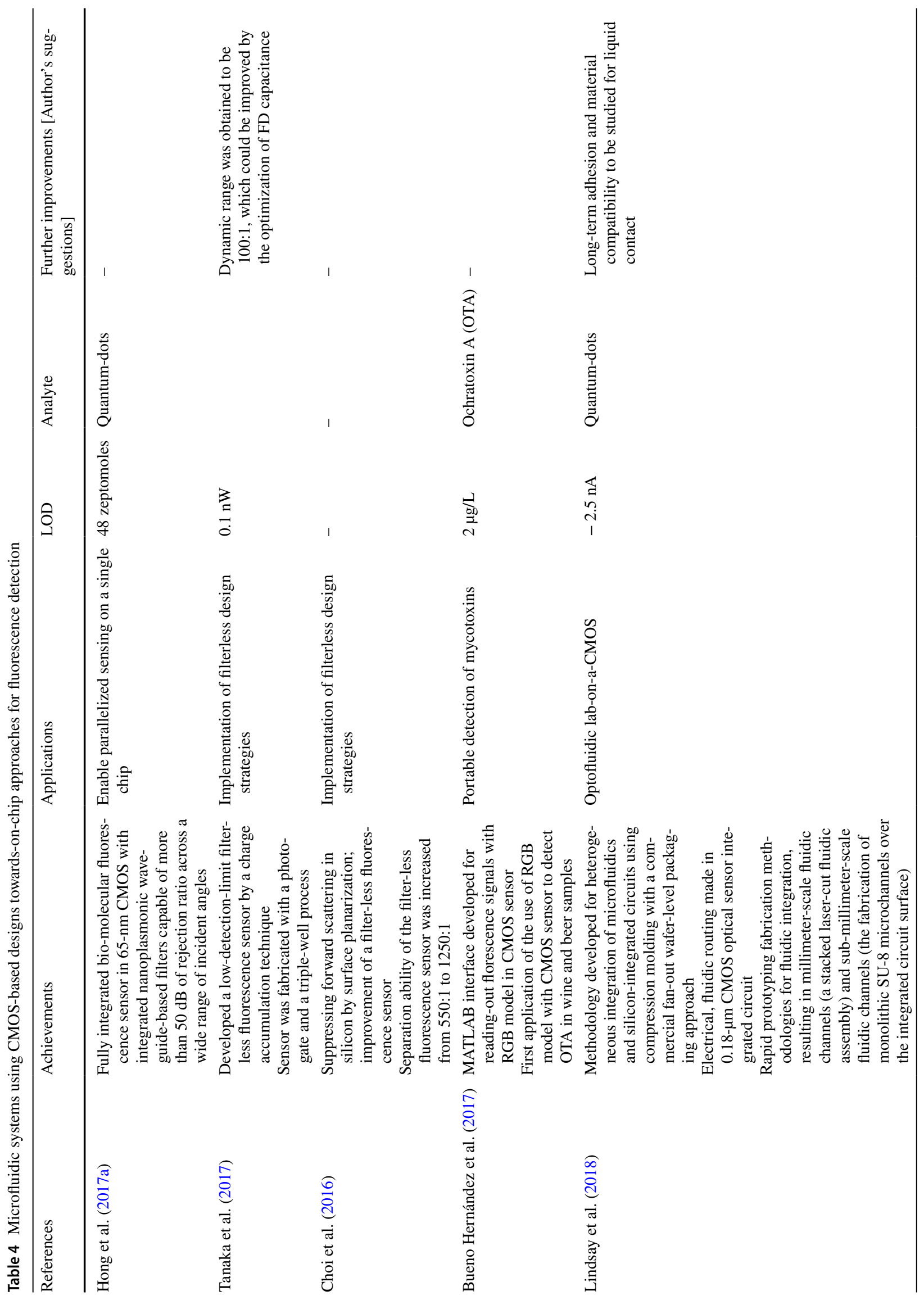




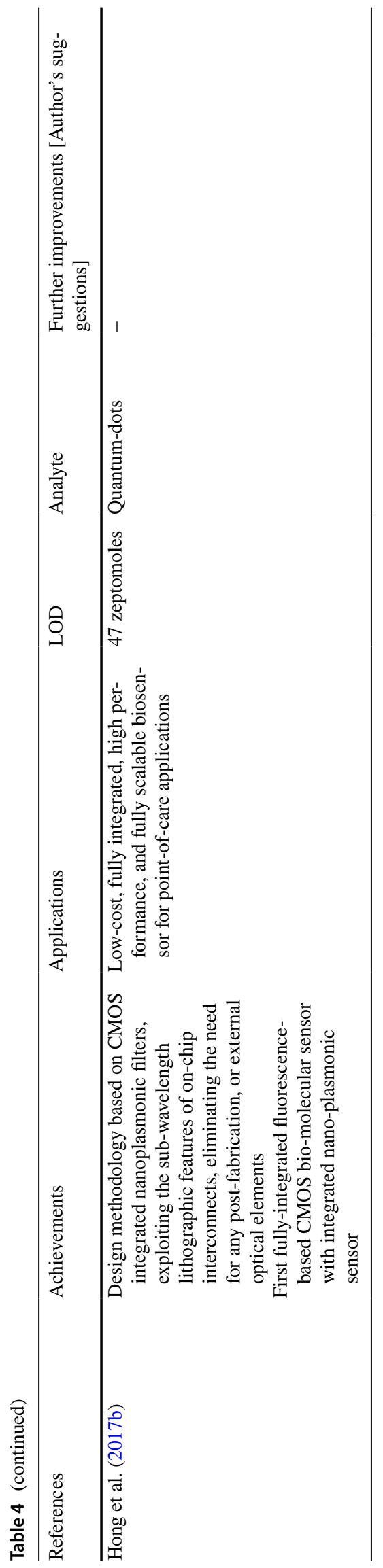

developed by implementing a $65 \mathrm{~nm}$ process, managing to diminish the noise by $50 \mathrm{~dB}$ for a large variety of incident angles. It comprised an assay platform, a sensor, and readout circuitry, making the integration of external optical components and any other post-fabrication techniques unnecessary, except for the light source that was a micro-laser diode or a LED. The optical fields could be manipulated in a controllable manner. Within the same team, the work of Lu et al. (2018) analyzed the possibility of photonic crystal integration on a CMOS sensor, exploiting optical physical unclonable functions (PUF) for a better management of the background noise.

In Varsanik and Bernstein (2013), a plasmonic resonator was designed, fabricated, and tested, proving both field enhancement and localization to nano dimensions. The proposed architecture enabled a solution for high-resolution and low-noise detection of fluorescence within an integrated microfluidic optical detection device. The microfluidic channel was built in a polymer on top of a glass substrate wafer. A diffused waveguide was embedded within the glass substrate, crossing the fluidic microchannel in the interrogation region. The width and the depth of the microchannel were $8 \mu \mathrm{m}$ and $200 \mathrm{~nm}$, respectively, enabling an extremely reduced interrogation volume and, by consequence, reduced noise. Finally, the system was capable to successfully detect $20 \mathrm{~nm}$ sized fluorescent particles.

CMOS-based fluorescence sensing field did not benefit from high-performance integrated optical filters until recently. This situation led to the implementation of either time-resolved techniques with synchronized sources (Samouda et al. 2015), or externally located optical filters and focusing optics (Lu et al. 2018). The above-mentioned methodologies, that were recently reported, enlarged the horizons toward more robust and compact fully-integrated solutions aiming to solve the chip-in-the-lab dilemma (Shakoor et al. 2018) with further improvements. The Internetof-Things (IoT) field of applications requires low-power and low-cost sensors, and here CMOS based fluorescence detection is expected to play a major role (Lu et al. 2018).

\subsection{Organic electronic-based designs}

Organic light-emitting diodes (OLEDs) and organic photon detectors (OPDs) are the subject of significant research efforts and continuous improvements (see Table 5) due to their multiple applications and advantages compared to their inorganic counterparts (Krujatz et al. 2016). Organic electronics introduced some unbeatable advantages, such as direct on-chip integration, easy emission and detection, and compatibility with flexible substrates. An OLED is a solid-state device composed of flexible thin films of organic molecules that emit light when subject to electricity, using less power than current available LEDs. 
(a)

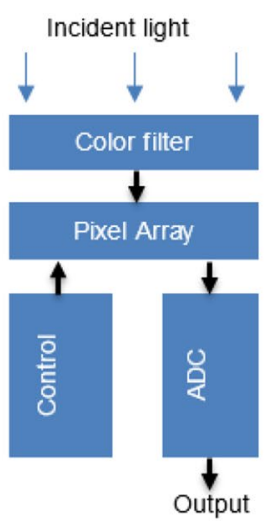

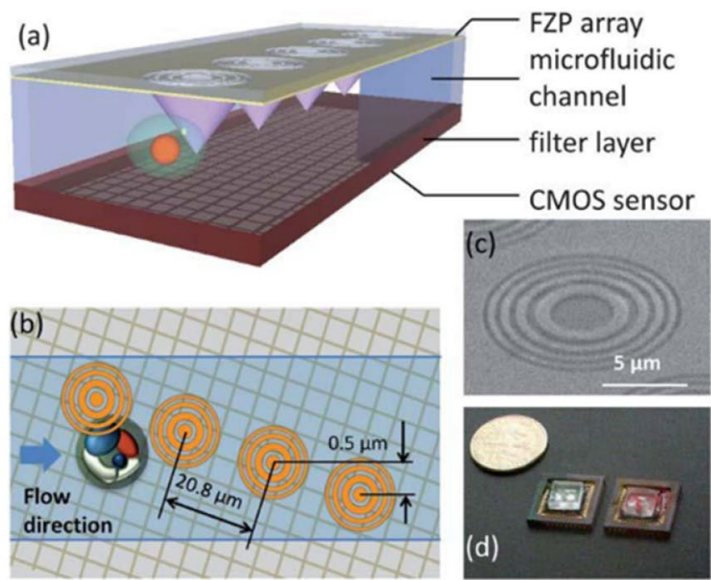

Fig. 8 a CMOS image sensors in-depth working principle. b (a) CMOS image sensor with thin layer filter coated on top, the microfluidic channel, and the Fresnel zone plate (FZP) array. (b) Top view schematic of the device with the FZP array configuration and its design parameters. (c) Scanning electron microscopy image of the fabricated Fresnel lens. (d) Images of the fabricated laboratory devices. Reproduced from (Pang et al. 2011), Copyright 2011, with permission from The Royal Society of Chemistry
Currently, the OLEDs are largely used in commercially available electronics (TVs, smartphones, etc.), being available in a large bandwidth emission spectrum. However, the OLEDs possess lower efficiencies (around $80 \mathrm{~lm} / \mathrm{W}$ ) than inorganic LEDs (higher than $200 \mathrm{~lm} / \mathrm{W}$ ) (Krujatz et al. 2016). For enhancing widespread OLED implementation, the PI-SCALE project (pi-scale.eu) aims to integrate existing European infrastructures into an "European flexible OLED pilot line", operating in an open access mode and serving customers with individual product designs, validation of upscaling concepts, and system-level flexible OLED integration. Comprehensive reviews of the latest achievements in OLEDs used for fluorescence sensing may be found in Williams et al. (2014), Jeong et al. (2015), Krujatz et al. (2016) and Yersin (2018). In Jansen-van Vuuren et al. (2016), recent achievements in the field of OPDs were described, while the last comprehensive review about the integration of fluorescence sensors using organic optoeletronics with microfluidics (see Fig. 9a) is presented by Lefèvre et al. (2015).

A fluorescence light detector combining both an organic electrochemical cell (OLEC) and an organic photodiode (OPD) within one microchip (see Fig. 9b, c) was introduced and tested by Shu et al. (2017). Moreover, linear polarizers were used as emission and excitation filters, enabling the possibility to detect fluorescent targets with emission and absorbing peaks very close to each other. The organic layers were manufactured using solution deposition processing, considerably decreasing the fabrications costs. The emission light peak of the OLEC was modified by choosing different light-emitting polymers, enabling multiplexing. The OLEC was used in pulsed mode to detect fluorescein amidite, reaching a $1 \mu \mathrm{M}$ limit of detection. The brightness of the OLEC was up to $2800 \mathrm{~cd} / \mathrm{m}^{2}$ at a driving voltage of
$50 \mathrm{~V}$. The brightness was not altered up to 10,000 pulses for a $30 \mathrm{~ms}$ pulse width, resulting in an autonomy of $10 \mathrm{~min}$. The proposed solution proved stability and high commercialization potential. Organic optoelectronics for building compact lab-on-a-chip applications was used by Jahns et al. (2017) who introduced two devices. For the first device, four $5 \mathrm{~mm}^{2}$ OLEDs and four $5 \mathrm{~mm}^{2}$ OPDs were manufactured separately on two $25 \times 25 \mathrm{~mm}^{2}$ glass substrates. A dichroic filter was used, to decrease the noise. The second device had a cylindrical form and used a reflection system, to facilitate the decrease of the noise and avoid additional filters.

A detailed description of the integration process of an organic optoelectronic system within a microfluidic platform has been presented in Poorahong et al. (2016). A system comprising a series of blue and green OLEDs, OPDs, and optical filters was designed, to develop a detection system for a PDMS multi-chambered structure with $9 \mu \mathrm{L}$ detection volumes. The $480 \mathrm{~nm}$ and $515 \mathrm{~nm}$ OLEDs, OPD, and optical filters were manufactured individually and then integrated within the microfluidic structure. The spectral width of the OLEDs was around $87-90 \mathrm{~nm}$. Their durability was tested by applying pulses of different voltages $(12 \mathrm{~V}$ for a blue OLED and $18 \mathrm{~V}$ for a green OLED) and after some tens of pulses, the emission intensity stayed constant. The limited lifetime of the organic materials, especially when high voltages are implied, limits the long-term usability. An innovative OPD manufacturing solution, enabling a 14,000 h lifetime under continuous operation was presented by Kielar et al. (2016).

Thin film transistor array technology, inspired by the flat panel display and X-ray image industry, is a new approach used to enable low-cost multi-biomarker detection. Smith et al. (2014) introduced a miniaturized fluorescence-based 


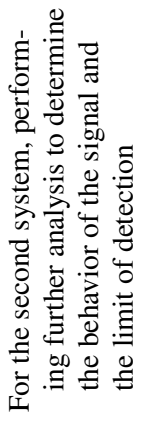

$\frac{2}{2}$
$\frac{3}{2}$
$\frac{0}{2}$
$\frac{0}{4}$

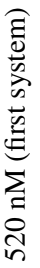

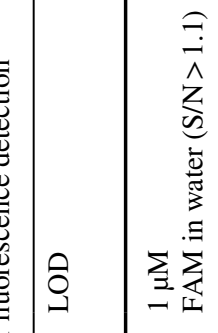

$\sum_{\substack{1 \\ 0}}$

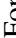

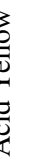

글

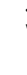

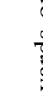

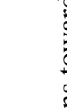

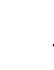




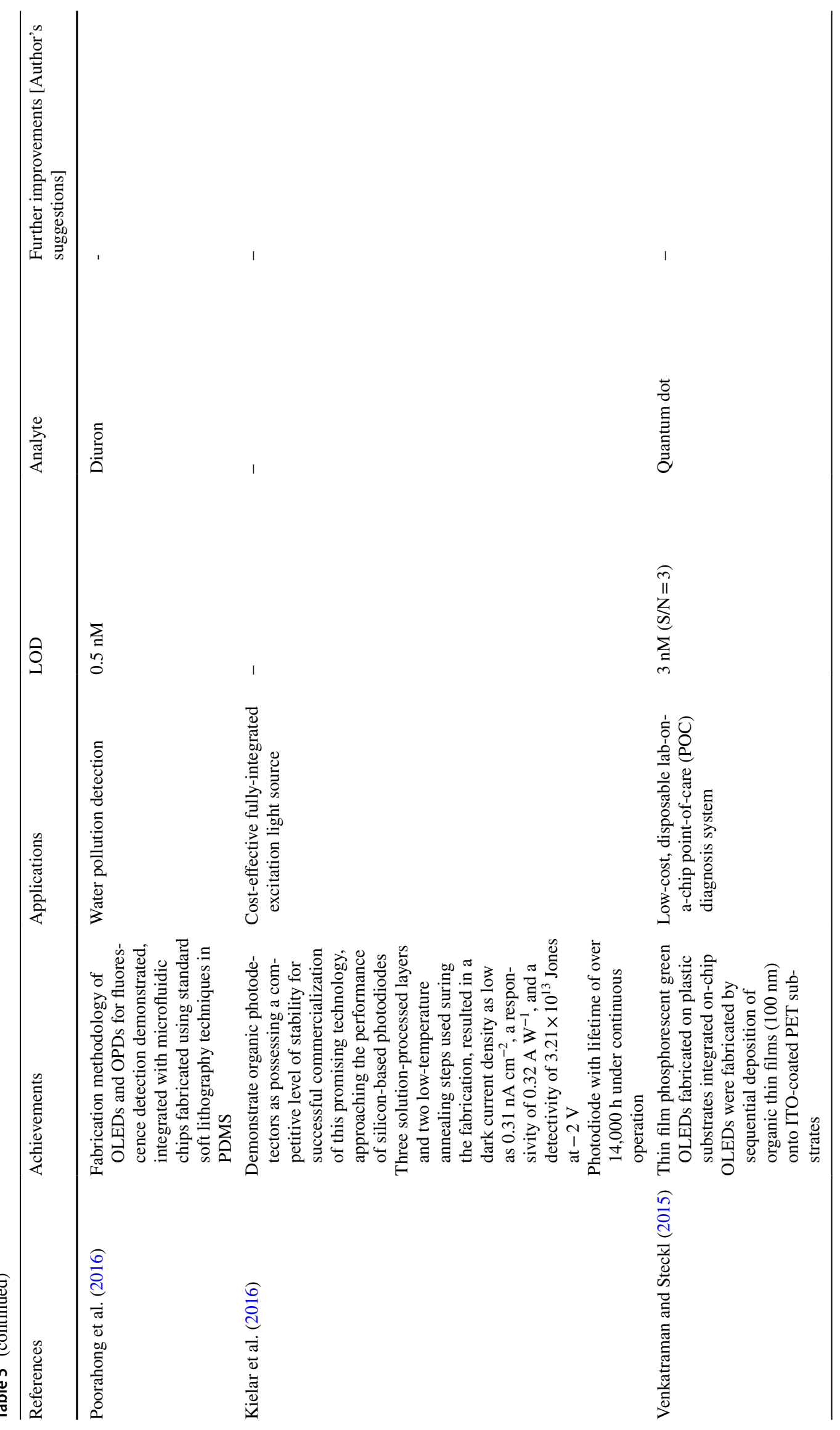


(a)

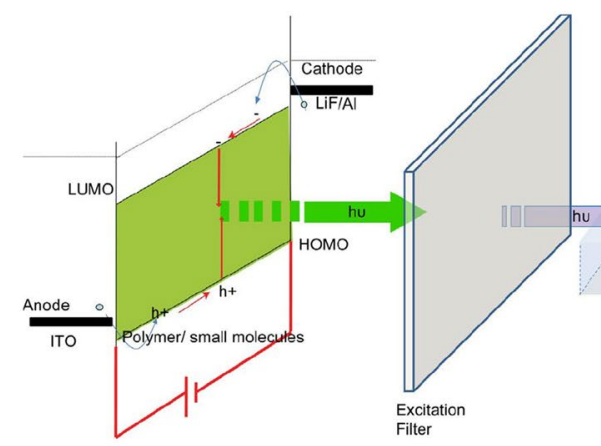

(a)

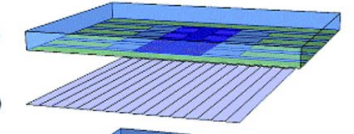

(c)

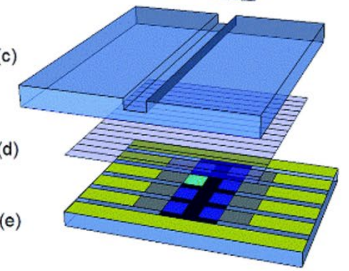

(b)

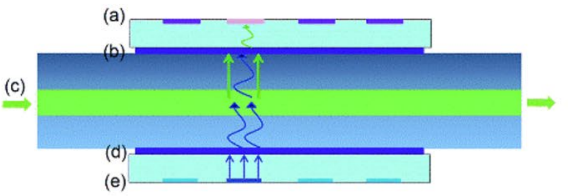

(c)

Fig. 9 a Generic illustration of the fluorescence optofluidic detection based on organic electronics. b Schematic representation of OLEC excitation and OPD detection system. (a) OPD. (b) Linear polarizer filter. (c) Glass microfluidic chip. (d) Blue OLEC. (e) fully-processed

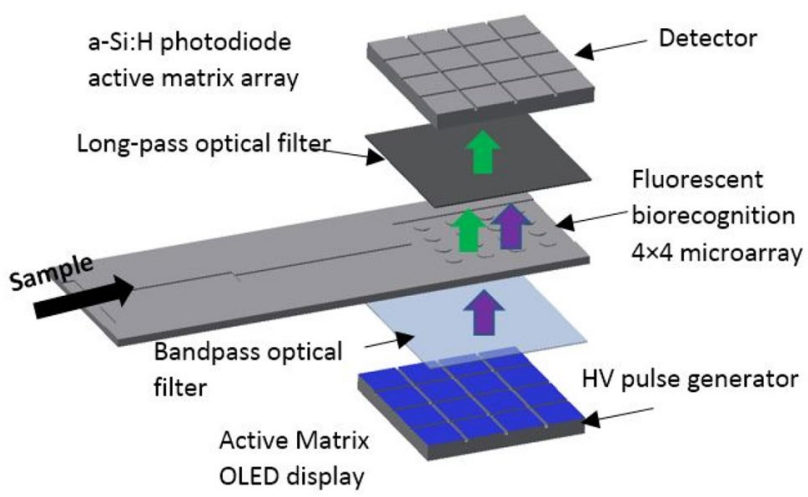

Fig. 10 Lab-on-a-chip sensing architecture based on OLED display and photodiode active matrix technology for point-of-use diagnosis of multiple diseases (Smith et al. 2014)

lab-on-a-chip sensing architecture (see Fig. 10) based on an OLED display and photodiode active matrix technology for point-of-use diagnosis of multiple diseases or pathogen markers in a cost-efficient disposable configuration. A straightforward concept, enabling a new approach using blue OLEC. c Fabricated optofluidic system. (a) Reproduced from (Lefèvre et al. 2015), Copyright 2015, with permission from Elsevier. (b) and (c) Reproduced from (Shu et al. 2017), Copyright 2017, with permission from The Royal Society of Chemistry

matrix active OLED and photodiode array technology for the multi-target analysis was implemented and tested. The $8 \times 8$ biorecognition array of 64 pixels based on this technology had an area lower than $2 \times 2 \mathrm{~mm}^{2}$ and was able to work with a $100 \mu \mathrm{L}$ volume of fluid. The array-based OLED was formed from multiple light-emitting elements (pixels) which were individually activated, to emit light at specific wavelengths and to enable multiple target detection. The same group proposed in Katchman et al. (2016) a high-density fluorescence, programmable, multiplexed recognition compact miniaturized device for point-of-care molecular diagnostics. The OLED technology was combined with protein microarray technology and $10 \mathrm{pg} / \mathrm{mL}$ limit of detection was achieved for human IgG.

\section{From current challenges to a possibly ideal concept: discussion}

It has been suggested that one of the reasons for which micro total analysis systems failed yet to cross the border from research to commercial application is the lack of a 
well-established design methodology (Volpatti and Yetisen 2014). Therefore, microfluidic devices still deploy complicated and sophisticated optical equipment to enhance fluorescence detection (Matteucci et al. 2015). This has partly been due to the reliability of detection still being too inadequate for meaningful results to be interpreted. Successful translation of micro-total-analysis systems from the laboratory to the market rely on the ability to integrate the detection components on-chip (Watts et al. 2012; Mohammed et al. 2015). The efficiency of the microfluidic processes taking place within the microchannels is often strictly related to the precise control of specific parameters, such as hydraulic diameter, and the memory effects (molecule adsorption within the walls structure affects the sensitivity and reliability of the sensor). Thus, working with fluids at microscale for sensing applications involves complexity. By consequence, there are still many issues which have to be solved regarding the repeatability, the portability, the ease of use and the sensitivity, the fabrication time, and the cost, before making these systems more advantageous than the classical world spread analysis systems (Wolfbeis 2013; Babikian et al. 2017). Currently, it can be emphasized that the optical and fluidic circuits were successfully miniaturized, while the entire system miniaturization is still challenging, more attention having to be focused on the system integration.

Some general parameters can be used for the quantification of the performances of a fluorescence-based sensor: fabrication cost, sensitivity, repeatability, multiplexing, auto-calibration, selectivity, response time, long-term stability, and autonomy. Among all the presented prototypes, some perform in one or several of these above-mentioned criteria, but none in all. While off-chip approaches achieved a high degree of maturity, very low prices and good performance, a multitude of methods are still tested for strategies towards on-chip integration. The microstructured optical fibers involving hollow core Bragg fibers refreshed the way optical fibers are implemented within fluorescence detection systems, proposing a design strategy integrating into one fiber the interrogation area, denoted the collector, the delivery channel, and the filter. The microfluidics-PCB concept managed to integrate microfluidics with optics and electronics in a monolithic manner, opening a promising development path. The printed circuit boards were used for both support of electronic components and microfluidic parts. Broad implementation of CMOS sensors, coupled with signal processing algorithms to filtrate and improve the sensitivity, could be observed in the recent literature. The CMOS technology coupled with contact sensing and time-correlated photon counting (TCSPC) emerged as a sound technology. It avoids usage of filtration and focusing optics. Nano-plasmonics coupled with CMOS technology managed to create enhanced fluorescence quantification on platforms with multiplexing capabilities. Organic electronics possess a huge potential, which is not fully exploited, both light emitting sources and photon detectors being already largely developed and commercially available. It offers unique advantages for fluorescence detection, such as flexibility of the emitting and detection layers, providing the possibility to develop flexible sensors. The tuning of different layers allows readout of a multitude of different wavelengths, enabling multiplex detection. Compared with LEDs, organic light-emitting diodes still possess lower efficiency, but recent achievements promise to improve this aspect (Krujatz et al. 2016).

It is obvious that further miniaturization strategies should focus on simplification of the traditional detection approach by identifying micro-fabrication and design architectures to replace/simplify the implementation of the intermediate light manipulation steps. A possible solution addressing this topic is the implementation of an advanced pattern recognition algorithm and/or selecting a more advanced photon detector, as some authors are suggesting (Babikian et al. 2017; Shin et al. 2017). Further development of fluorescence optical sensors should envisage strategies involving all the elements as they are illustrated in Fig. 11. This is mandatory

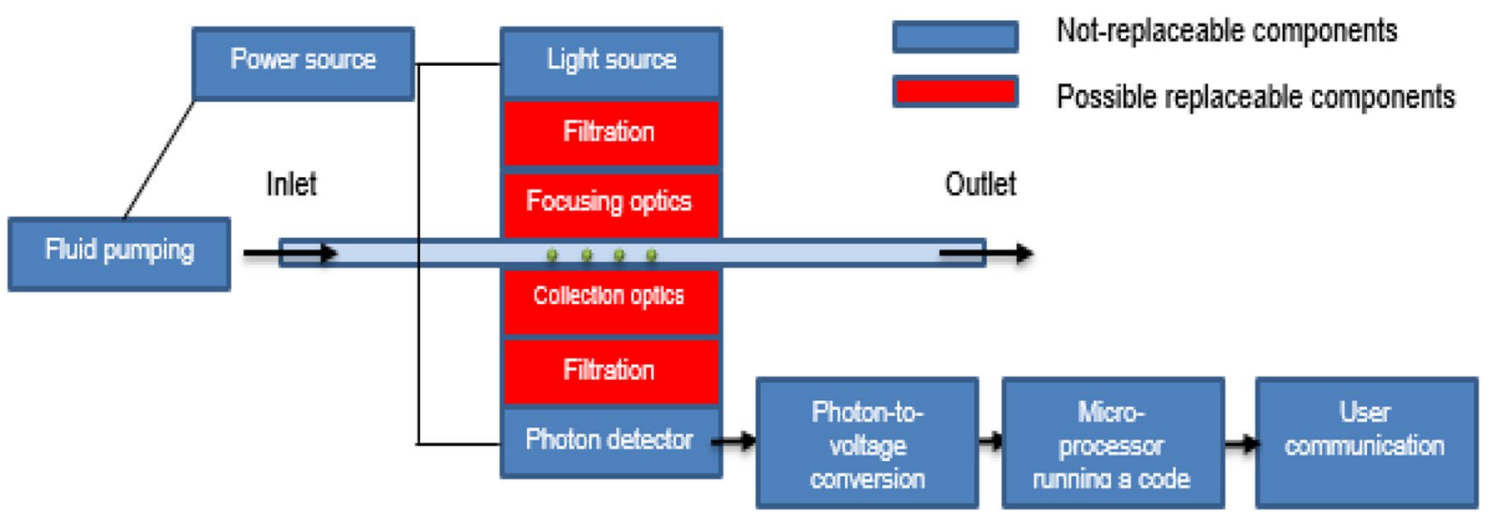

Fig. 11 Fluorescence sensing: general integration scheme 
for solving the chip-in-the-lab dilemma that refers to the lack of portability, operation simplicity, and reliability outside of the laboratory of the majority of the miniaturized analytical systems.

Once the miniaturization dream of chemical and biological sensing would be achieved, the following step is easy to be anticipated. Large sensing networks coupled with AI and IoT would allow rapid and in-time measurement of the presence of specific indicators identified in small amounts of body fluids, better monitoring pollution, and many other applications.

Acknowledgements Open Access funding provided by Projekt DEAL. The work in this article has received funding from the European Union's Horizon 2020 research and innovation programme under the Marie Sklodowska-Curie Grant agreement no. 643095.

Author contributions All authors contributed to this review article. DM had the idea of the article, performed the literature search, and data analysis. The first draft of the manuscript was written by DM and all authors commented, critically revised and give suggestions on previous versions of the manuscript. All authors read and approved the final manuscript.

Funding The work in this article has received funding from the European Union's Horizon 2020 research and innovation programme under the Marie Sklodowska-Curie grant agreement no. 643095.

Availability of data and material (data transparency) All data generated or analysed during this study are included in this published article.

\section{Compliance with ethical standards}

Conflict of interest The authors declare that they have no conflict of interest.

Open Access This article is licensed under a Creative Commons Attribution 4.0 International License, which permits use, sharing, adaptation, distribution and reproduction in any medium or format, as long as you give appropriate credit to the original author(s) and the source, provide a link to the Creative Commons licence, and indicate if changes were made. The images or other third party material in this article are included in the article's Creative Commons licence, unless indicated otherwise in a credit line to the material. If material is not included in the article's Creative Commons licence and your intended use is not permitted by statutory regulation or exceeds the permitted use, you will need to obtain permission directly from the copyright holder. To view a copy of this licence, visit http://creativecommons.org/licenses/by/4.0/.

\section{References}

Ackermann TN, Dietvorst J, Sanchis A et al (2016a) Modular optofluidic systems (MOPS). In: SPIE BioPhotonics Australasia. p $100131 \mathrm{C}$

Ackermann TN, Giménez-Gómez P, Muñoz-Berbel X, Llobera A (2016) Plug and measure-a chip-to-world interface for photonic lab-on-a-chip applications. Lab Chip 16:3220-3226. https://doi. org/10.1039/c6lc00462h
Babikian S, Li GP, Bachman M (2017) A digital signal processingassisted microfluidic PCB for on-chip fluorescence detection. IEEE Trans Compon Packag Manuf Technol 7:846-854. https ://doi.org/10.1109/TCPMT.2017.2691673

Balsam J, Ossandon M, Bruck HA, Rasooly A (2012) Modeling and design of micromachined optical Söller collimators for lensless CCD-based fluorometry. Analyst 137:5011-5017. https://doi. org/10.1039/c2an35729a

Bates KE, Lu H (2016) Optics-integrated microfluidic platforms for biomolecular analyses. Biophys J 110:1684-1697. https://doi. org/10.1016/j.bpj.2016.03.018

Bauch M, Toma K, Toma M et al (2014) Plasmon-enhanced fluorescence biosensors: a review. Plasmonics 9:781-799. https://doi. org/10.1007/s11468-013-9660-5

Benito-Peña E, Valdés MG, Glahn-Martínez B, Moreno-Bondi MC (2016) Fluorescence based fiber optic and planar waveguide biosensors: a review. Anal Chim Acta 943:17-40. https://doi. org/10.1016/j.aca.2016.08.049

Berner M, Hilbig U, Schubert MB, Gauglitz G (2017) Laser-induced fluorescence detection platform for point-of-care testing. Meas Sci Technol. https://doi.org/10.1088/1361-6501/aa7810

Bhargava KC, Thompson B, Malmstadt N (2014) Discrete elements for 3D microfluidics. Proc Natl Acad Sci USA 111:1501315018. https://doi.org/10.1073/pnas.1414764111

Bhattacharya B, Singha S, Basu S (2017) Fluorescent nanosensors: rapid tool for detection of food contaminants. Elsevier Inc, Amsterdam

Boppart SA, Richards-Kortum R (2014) Point-of-care and pointof-procedure optical imaging technologies for primary care and global health. Sci Transl Med. https://doi.org/10.1126/scitr anslmed.3009725

Brammer M, Mappes T (2014) Modular platforms for optofluidic systems. Optofluidics. https://doi.org/10.2478/optof-2013-0001

Bueno Hernández D, Mishra RK, Muñoz R, Marty JL (2017) Low cost optical device for detection of fluorescence from Ochratoxin A using a CMOS sensor. Sens Actuators B Chem 246:606-614. https://doi.org/10.1016/j.snb.2017.02.097

Calvé SL, Trocquet C, Bernhardt P (2017) A novel microfluidic formaldehyde microanalyser for continuous real-time monitoring in Indoor air: analytical development and validation. Multidisciplinary Digital Publishing Institute, Barcelona, p 740

Chen D, Yang T-J, Wu J-J et al (2008) Band-rejection fiber filter and fiber sensor based on a Bragg fiber of transversal resonant structure. Opt Express 16:16489. https://doi.org/10.1364/ oe.16.016489

Chen Y-F, Jiang L, Mancuso M et al (2012) Nanoscale optofluidic opportunities in global health, food, water and energy. Nanoscale. https://doi.org/10.1039/c2nr30859b

Chen S, Hao R, Zhang Y, Yang H (2019) Optofluidics in bio-imaging applications. Photonics Res 7:532. https://doi.org/10.1364/ prj.7.000532

Choi YJ, Takahashi K, Matsuda M et al (2016) Filter-less fluorescence sensor with high separation ability achieved by the suppression of forward-scattered light in silicon. Jpn J Appl Phys. https://doi.org/10.7567/JJAP.55.04EM10

Dandin M, Abshire P, Smela E (2007) Optical filtering technologies for integrated fluorescence sensors. Lab Chip 7:955-977. https ://doi.org/10.1039/b704008c

Datta-Chaudhuri T, Smela E, Abshire PA (2016) System-on-chip considerations for heterogeneous integration of CMOS and fluidic bio-interfaces. IEEE Trans Biomed Circuits Syst 10:11291142. https://doi.org/10.1109/TBCAS.2016.2522402

Ertman S, Lesiak P, Woli TR, Paper I (2017) Optofluidic photonic crystal fiber-based sensors. J Light Technol 35:3399-3405. https://doi.org/10.1109/JLT.2016.2596540 
Fang XX, Li HY, Fang P et al (2016) A handheld laser-induced fluorescence detector for multiple applications. Talanta 150:135141. https://doi.org/10.1016/j.talanta.2015.12.018

Geddes CD (2017) Surface plasmon enhanced, coupled and controlled fluorescence. Wiley, New Jersey

Ghosh KK, Burns LD, Cocker ED et al (2011) Miniaturized integration of a fluorescence microscope. Nat Methods 8:871-878. https://doi.org/10.1038/nmeth.1694

Gründler P (2007) Chemical sensors: an introduction for scientists and engineers. Springer, Berlin

Gu Y, Bragheri F, Valentino G et al (2015) Ferrofluid-based optofluidic switch using femtosecond laser-micromachined waveguides. Appl Opt 54:1420. https://doi.org/10.1364/ao.54.00142 0

Guduru SSK, Scotognella F, Chiasera A et al (2016) Highly integrated lab-on-a-chip for fluorescence detection. Opt Eng 55:097102. https://doi.org/10.1117/1.oe.55.9.097102

Harmon DM, Chen CF, Halford JH (2018) Portable fluorescence detection platform with integrating sphere. In: Proceedings of the annual international conference of the IEEE engineering in medicine and biology society, EMBS. pp 2889-2892

Hatch AC, Ray T, Lintecum K, Youngbull C (2014) Continuous flow real-time PCR device using multi-channel fluorescence excitation and detection. Lab Chip 14:562-568. https://doi.org/10.1039/ c31c51236c

He F, Liao Y, Lin J et al (2014) Femtosecond laser fabrication of monolithically integrated microfluidic sensors in glass. Sensors (Switzerland) 14:19402-19440. https://doi.org/10.3390/s141019402

Hierlemann A, Brand O, Hagleitner C, Baltes H (2003) Microfabrication techniques for chemical/biosensors. Proc IEEE 91:839-863

Hong L, Li H, Yang H, Sengupta K (2017) Fully integrated fluorescence biosensors on-chip employing multi-functional nanoplasmonic optical structures in CMOS. IEEE J Solid State Circuits 52:2388-2406. https://doi.org/10.1109/JSSC.2017.2712612

Hong L, Lu X, Sengupta K (2017b) Nano-optical systems in CMOS. In: Midwest symposium on circuits and systems. pp 906-909

Huang Y, Xu Y, Yariv A (2004) Fabrication of functional microstructured optical fibers through a selective-filling technique. Appl Phys Lett 85:5182-5184. https://doi.org/10.1063/1.1828593

Jahns S, Iwers AFK, Balke J, Gerken M (2017) Organic optoelectronics for lab-on-chip fluorescence detection. In: Technisches Messen. pp S48-S51

Jang DH, Yoo JC (2013) Miniaturized fluorometer based on total internal reflector and condensing mirror. J Opt Soc Korea 17:81-85. https://doi.org/10.3807/JOSK.2013.17.1.081

Jansen-van Vuuren RD, Armin A, Pandey AK et al (2016) Organic photodiodes: the future of full color detection and image sensing. Adv Mater. https://doi.org/10.1002/adma.201505405

Jeong H, Shin H, Lee J et al (2015) Recent progress in the use of fluorescent and phosphorescent organic compounds for organic light-emitting diode lighting. J Photonics Energy. https://doi. org/10.1117/1.jpe.5.057608

Joseph KAJ, Haque M, Ho S et al (2017) Femtosecond laser directwrite of optofluidics in polymer-coated optical fiber. In: Proceedings of SPIE 10094, Frontiers in Ultrafast Optics: Biomedical, Scientific, and Industrial Applications XVII. p 1009414

Kang D, Gai B, Thompson B et al (2016) Flexible opto-fluidic fluorescence sensors based on heterogeneously integrated microVCSELs and silicon photodiodes. ACS Photonics 6:912-918. https://doi.org/10.1021/acsphotonics.6b00080

Katchman BA, Smith JT, Obahiagbon U et al (2016) Application of flat panel OLED display technology for the point-of-care detection of circulating cancer biomarkers. Sci Rep 6:1-11. https://doi. org/10.1038/srep29057
Kielar M, Dhez O, Pecastaings G et al (2016) Long-term stable organic photodetectors with ultra low dark currents for high detectivity applications. Sci Rep 6:1-11. https://doi.org/10.1038/srep39201

Kilic T, Navaee F, Stradolini F et al (2018) Organs-on-chip monitoring: sensors and other strategies. Microphysiol Syst. https://doi. org/10.21037/mps.2018.01.01

Krujatz F, Hild F, Fehse K, Jahnel M (2016) Exploiting the potential of OLED-based photo-organic sensors for biotechnological applications. Chem Sci J. https://doi.org/10.4172/2150-3494.1000134

Kuswandi B, Nuriman HJ, Verboom W (2007) Optical sensing systems for microfluidic devices: a review. Anal Chim Acta 601:141-155

Lee Y, Kim B, Oh I, Choi S (2018) Optofluidic modular blocks for ondemand and open-source prototyping of microfluidic systems. Small. https://doi.org/10.1002/smll.201802769

Lefèvre F, Juneau P, Izquierdo R (2015) Integration of fluorescence sensors using organic optoelectronic components for microfluidic platform. Sens Actuators B Chem 221:1314-1320. https://doi. org/10.1016/j.snb.2015.07.077

Li J, Nallappan K (2019) Optimization of hollow-core photonic Bragg fibers towards practical sensing implementations. Opt Mater Express 9:1640. https://doi.org/10.1364/ome.9.001640

Lindsay M, Member S, Bishop K et al (2018) Heterogeneous integration of CMOS sensors and fluidic networks using wafer-level molding. IEEE Trans Biomed Circuits Syst 12:1046-1055. https ://doi.org/10.1109/TBCAS.2018.2845867

Llobera A, Juvert J, González-Fernández A et al (2015) Biofunctionalized all-polymer photonic lab on a chip with integrated solid-state light emitter. Light Sci Appl. https://doi.org/10.1038/ lsa. 2015.44

Lu X, Hong L, Sengupta K (2018) CMOS optical PUFs using noiseimmune process-sensitive photonic crystals incorporating passive variations for robustness. IEEE J Solid State Circuits 53:2709-2721. https://doi.org/10.1109/JSSC.2018.2850941

Măriuța D, Baldas L, Colin S et al (2019) Microfluidic sensing of airborne formaldehyde : towards on-chip integration (oral presentation and poster and proceedings with peer review ) To cite this version : HAL Id : hal-02365974

Măriuța D, Baldas L, Colin S et al (2020) Prototyping a microfluidic sensor for real-time detection of airborne formaldehyde. Int J Chem Eng Appl 11:23-28. https://doi.org/10.18178/ijcea .2020.11.1.774

Matteucci M, Triches M, Nava G et al (2015) Fiber-based, injection-molded optofluidic systems: improvements in assembly and applications. Micromachines 6:1971-1983. https://doi. org/10.3390/mi6121468

Measor P, Phillips BS, Chen A et al (2011) Tailorable integrated optofluidic filters for biomolecular detection. Lab Chip 11:899-904. https://doi.org/10.1039/c0lc00496k

Minzioni P, Osellame R, Sada C et al (2017) Roadmap for optofluidics. J Opt (United Kingdom) 19:93003. https://doi.org/10.1088/20408986/aa783b

Mohammed MI, Haswell S, Gibson I (2015) Lab-on-a-chip or chip-ina-lab: challenges of commercialization lost in translation. Proced Technol 20:54-59. https://doi.org/10.1016/j.protcy.2015.07.010

Nakazawa H, Yamasaki K, Takahashi K et al (2011) A filter-less multiwavelength fluorescence detector. In: 2011 16th International solid-state sensors, actuators microsystems conference TRANSDUCERS'11, pp 100-103. https://doi.org/10.1109/TRANS DUCERS.2011.5969147

Ngernsutivorakul T, Cipolla CM, Dugan CE et al (2017) Design and microfabrication of a miniature fiber optic probe with integrated lenses and mirrors for Raman and fluorescence measurements. Anal Bioanal Chem 409:275-285. https://doi.org/10.1007/s0021 6-016-9999-5

Nittala PVK, Sen P (2018) Scaling a fluorescent detection system by polymer-assisted 3-D integration of heterogeneous dies. $\mathrm{J}$ 
Microelectromech Syst 27:896-909. https://doi.org/10.1109/ JMEMS.2018.2856935

Novo P, Chu V, Conde JP (2014) Integrated fluorescence detection of labeled biomolecules using a prism-like PDMS microfluidic chip and lateral light excitation. Lab Chip 14:1991-1995. https://doi. org/10.1039/c4lc00241e

Obahiagbon U, Smith JT, Zhu M et al (2018) A compact, low-cost, quantitative and multiplexed fluorescence detection platform for point-of-care applications. Biosens Bioelectron 117:153-160. https://doi.org/10.1016/j.bios.2018.04.002

Østergaard PF, Lopacinska-Jørgensen J, Pedersen JN et al (2015) Optical mapping of single-molecule human DNA in disposable, mass-produced all-polymer devices. J Micromech Microeng. https://doi.org/10.1088/0960-1317/25/10/105002

Özbakır Y, Jonas A, Kiraz A, Erkey C (2017) Aerogels for optofluidic waveguides. Micromachines. https://doi.org/10.3390/mi8040098

Paiè P, Bragheri F, Claude T, Osellame R (2017) Optofluidic light modulator integrated in lab-on-a-chip. Opt Express 25:7313. https://doi.org/10.1364/oe.25.007313

Pan JZ, Fang P, Fang XX et al (2018) A low-cost palmtop high-speed capillary electrophoresis bioanalyzer with laser induced fluorescence detection. Sci Rep 8:1-11. https://doi.org/10.1038/s4159 8-018-20058-0

Pang S, Han C, Lee LM, Yang C (2011) Fluorescence microscopy imaging with a Fresnel zone plate array based optofluidic microscope. Lab Chip 21:3698-3702. https://doi.org/10.1039/c1lc2 $0654 \mathrm{k}$

Papageorgiou EP, Boser BE, Anwar M (2017) Chip-scale fluorescence imager for in vivo microscopic cancer detection. IEEE Symposium on VLSI Circuits. Digest of Technical Papers. Kyoto, Japan, pp C106-C107

Parks JW, Olson MA, Kim J et al (2014) Integration of programmable microfluidics and on-chip fluorescence detection for biosensing applications. Biomicrofluidics. https://doi.org/10.1063/1.48972 26

Persichetti G, Grimaldi IA, Testa G, Bernini R (2017) Multifunctional optofluidic lab-on-chip platform for Raman and fluorescence spectroscopic microfluidic analysis. Lab Chip 17:2631-2639. https://doi.org/10.1039/c7lc00460e

Pfeiffer SA, Nagl S (2015) Microfluidic platforms employing integrated fluorescent or luminescent chemical sensors: a review of methods, scope and applications. Methods Appl Fluoresc. https://doi. org/10.1088/2050-6120/3/3/034003

Pires NMM, Dong T, Hanke U, Hoivik N (2014) Recent developments in optical detection technologies in lab-on-a-chip devices for biosensing applications. Sensors (Switzerland) 14:15458-15479. https://doi.org/10.3390/s140815458

Poorahong S, Lefevre F, Perron MC et al (2016) Integration of optical and electrochemical sensors on a microfluidic platform using organic optoelectronic components and silver nanowires. In: Proceedings of the annual international conference of the IEEE engineering in medicine and biology society (EMBS) 2016-Octob: pp 3002-3005. https://doi.org/10.1109/EMBC.2016.7591361

Rezende GC, Le Calvé S, Brandner JJ, Newport D (2019) Chemical micro photoionization detectors. Sens Actuators B Chem 287:86-94. https://doi.org/10.1016/j.snb.2019.01.072

Ricciardi A, Crescitelli A, Vaiano P et al (2015) Lab-on-fiber technology: a new vision for chemical and biological sensing. Analyst 140:8068-8079

Ricciardi S, Frascella F, Angelini A et al (2015) Optofluidic chip for surface wave-based fluorescence sensing. Sens Actuators B Chem 215:225-230. https://doi.org/10.1016/j.snb.2015.03.063

Ríos Á, Zougagh M, Avila M (2012) Miniaturization through lab-ona-chip: Utopia or reality for routine laboratories? A review. Anal Chim Acta 740:1-11. https://doi.org/10.1016/j.aca.2012.06.024
Robbins H, Sumitomo K, Tsujimura N, Kamei T (2018) Integrated thin film Si fluorescence sensor coupled with a GaN microLED for microfluidic point-of-care testing. J Micromech Microeng. https ://doi.org/10.1088/1361-6439/aa9e6d

Rodríguez-Ruiz I, Masvidal-Codina E, Ackermann TN, Llobera A (2015) Photonic lab-on-chip (PhLOC) for enzyme-catalyzed reactions in continuous flow. Microfluid Nanofluidics 18:12771286. https://doi.org/10.1007/s10404-014-1526-4

Rodríguez-Ruiz I, Ackermann TN, Muñoz-Berbel X, Llobera A (2016) Photonic lab-on-a-chip: integration of optical spectroscopy in microfluidic systems. Anal Chem 88:6630-6637. https://doi. org/10.1021/acs.analchem.6b00377

Rodríguez-Ruiz I, Teychené S, Van Pham N et al (2017) Broadcasting photonic lab on a chip concept through a low cost manufacturing approach. Talanta 170:180-184. https://doi.org/10.1016/j.talan ta.2017.04.010

Ryu G, Huang J, Hofmann O et al (2011) Highly sensitive fluorescence detection system for microfluidic lab-on-a-chip. Lab Chip 11:1664-1670. https://doi.org/10.1039/c0lc00586j

Samouda F, Colin S, Barrot C et al (2015) Micro molecular tagging velocimetry for analysis of gas flows in mini and micro systems. Microsyst Technol 21:527-537. https://doi.org/10.1007/s0054 2-013-1971-0

Serhatlioglu M, Elbuken C, Ortac B, Solmaz ME (2017) Femtosecond laser fabrication of fiber based optofluidic platform for flow cytometry applications. In: Proceedings of SPIE 10058, optical fibers and sensors for medical diagnostics and treatment applications XVII 100580I:100580I. https://doi.org/10.1117/12.22520 92

Shakoor A, Cheah BC, Al-rawhani MA et al (2018) CMOS nanophotonic sensor with integrated readout system. IEEE Sens J 18:9188-9194. https://doi.org/10.1109/JSEN.2018.2870255

Shang L, Zheng K (2017) Design of hollow core Bragg fibers for a compact fluorescence sensing scheme. IEEE Photonics J. https ://doi.org/10.1109/JPHOT.2017.2677582

Shao L (2018) Optofluidics in microstructured optical fibers. Micromachines 9:145. https://doi.org/10.3390/mi9040145

Sharma MK, Göstl R, Frijns AJH et al (2018) A fluorescent microoptofluidic sensor for in-line ion selective electrolyte monitoring. IEEE Sens J 18:3946-3951. https://doi.org/10.1109/ JSEN.2018.2816986

Shin YH, Barnett JZ, Song E et al (2015) A portable fluorescent sensor for on-site detection of microalgae. Microelectron Eng 144:6-11. https://doi.org/10.1016/j.mee.2015.01.005

Shin Y-H, Barnett JZ, Gutierrez-Wing MT et al (2017) A portable fluorescent sensing system using multiple LEDs. Microfluid BioMEMS Med Microsyst XV. https://doi.org/10.1117/12.22612 92

Shu Z, Kemper F, Beckert E et al (2017) Highly sensitive on-chip fluorescence sensor with integrated fully solution processed organic light sources and detectors. RSC Adv 7:26384-26391. https:// doi.org/10.1039/c7ra03841k

Si Hadj Mohand H, Frezzotti A, Brandner JJ et al (2017) Molecular tagging velocimetry by direct phosphorescence in gas microflows: correction of Taylor dispersion. Exp Therm Fluid Sci 83:177190. https://doi.org/10.1016/j.expthermflusci.2017.01.002

Siudzinska A, Miszczuk A, Marczak J, Komorowska K (2017) Fluorescent sensing with Fresnel microlenses for optofluidic systems. Opt Eng. https://doi.org/10.1117/1.OE.56.5.057106

Smith JT, Katchman BA, Lee YK et al (2014) Disposable point-ofuse optical biosensor for multiple biomarker detection. In: IEEE 2014 Biomedical circuits and systems conference (BioCAS) 2014-proceedings, pp 268-271. https://doi.org/10.1109/BioCA S.2014.6981714 
Song C, Tan SH (2017) A perspective on the rise of optofluidics and the future. Micromachines 8:1-17. https://doi.org/10.3390/mi805 0152

Song C, Nguyen NT, Tan SH (2017) Toward the commercialization of optofluidics. Microfluid Nanofluidics 21:1-16. https://doi. org/10.1007/s10404-017-1978-4

Sudhakaran S (2020) What is the difference between CIE LAB, CIE RGB, CIE xyY and CIE XYZ? https://wolfcrow.com/what-isthe-difference-between-cie-lab-cie-rgb-cie-xyy-and-cie-xyz/. Accessed 20 Dec 2019

Sugioka K, Cheng Y (2012) Femtosecond laser processing for optofluidic fabrication. Lab Chip 12:3576-3589. https://doi. org/10.1039/c2lc40366h

Sugioka K, Xu J, Wu D et al (2014) Femtosecond laser 3D micromachining: a powerful tool for the fabrication of microfluidic, optofluidic, and electrofluidic devices based on glass. Lab Chip 14:3447-3458. https://doi.org/10.1039/c4lc00548a

Tanaka K, Choi YJ, Moriwaki Y et al (2017) Improvements of lowdetection-limit filter-free fluorescence sensor developed by charge accumulation operation. Jpn J Appl Phys 56:2-7

Torres-mapa ML, Singh M, Simon O et al (2019) Fabrication of a monolithic lab-on-a-chip platform with integrated hydrogel waveguides for chemical sensing. Sensors (Switzerland) 19:4333. https://doi.org/10.3390/s19194333

Varsanik JS, Bernstein JJ (2013) Integrated optic/nanofluidic fluorescent detection device with plasmonic excitation. J Micromech Microeng. https://doi.org/10.1088/0960-1317/23/9/095017

Venkatraman V, Steckl AJ (2015) Integrated OLED as excitation light source in fluorescent lateral flow immunoassays. Biosens Bioelectron 74:150-155. https://doi.org/10.1016/j.bios.2015.06.049

Volpatti LR, Yetisen AK (2014) Commercialization of microfluidic devices. Trends Biotechnol 32:347-350. https://doi. org/10.1016/j.tibtech.2014.04.010

Watts BR, Zhang Z, Xu C-Q et al (2012) Integration of optical components on-chip for scattering and fluorescence detection in an optofluidic device. Biomed Opt Express 3:2784. https://doi. org/10.1364/boe.3.002784

Wei L, Yan W, Ho D (2017) Recent advances in fluorescence lifetime analytical microsystems: contact optics and CMOS time-resolved electronics. Sensors (Switzerland). https://doi.org/10.3390/s1712 2800

Wessels L, Raad H (2016) Recent advances in point of care diagnostic tools: a review. Am J Eng Appl Sci 9:1088-1095. https://doi. org/10.3844/ajeassp.2016.1088.1095

Williams G, Backhouse C, Aziz H (2014) Integration of organic light emitting diodes and organic photodetectors for lab-on-a-chip bio-detection systems. Electron 3:43-75. https://doi.org/10.3390/ electronics 3010043

Wolfbeis OS (2013) Editorial: Probes, sensors, and labels: why is real progress slow? Angew Chem Int Ed 52:9864-9865. https://doi. org/10.1002/anie.201305915

Wu J, Gu M (2011) Microfluidic sensing: state of the art fabrication and detection techniques. J Biomed Opt 16:080901. https://doi. org/10.1117/1.3607430

Yang H, Gijs MAM (2018) Micro-optics for microfluidic analytical applications. Chem Soc Rev 47:1391-1458. https://doi. org/10.1039/c5cs00649j

Yang X, Yuan T, Yue G et al (2015) Optofluidic integrated in-fiber fluorescence online optical fiber sensor. Sens Actuators B Chem 215:345-349. https://doi.org/10.1016/j.snb.2015.04.003

Yeh P, Yeh N, Lee CH, Ding TJ (2017) Applications of LEDs in optical sensors and chemical sensing device for detection of biochemicals, heavy metals, and environmental nutrients. Renew Sustain Energy Rev 75:461-468. https://doi.org/10.1016/j. rser.2016.11.011

Yersin H (2018) Highly efficient OLEDs: materials based on thermally activated delayed fluorescence. Wiley-VCH Verlag $\mathrm{GmbH} \& \mathrm{Co}$. $\mathrm{KGaA}$, New york

Yue W, Zhang L, Guo Z et al (2015) Portable and modularized fluorometer based on optical fiber. Ninth Int Symp Precis Eng Meas Instrum. https://doi.org/10.1117/12.2182022

Zhang YS, Ribas J, Nadhman A et al (2015) A cost-effective fluorescence mini-microscope for biomedical applications. Lab Chip 15:3661-3669. https://doi.org/10.1039/c5lc00666j

Zhang Y, Watts BR, Guo T et al (2016) Optofluidic device based microflow cytometers for particle/cell detection: a review. Micromachines 7:1-21. https://doi.org/10.3390/mi7040070

Zhao Z, Luan W, Yin S, Brandner JJ (2015) Metal crack propagation monitoring by photoluminescence enhancement of quantum dots. Appl Opt 54:6498-6501. https://doi.org/10.1364/ao.54.006498

Zhu H, Isikman SO, Mudanyali O et al (2013) Optical imaging techniques for point-of-care diagnostics. Lab Chip 13:51-67. https ://doi.org/10.1039/c2lc40864c

$\mathrm{Zi} \mathrm{C}$, Shi J, Tai Y et al (2016) Design of portable time-resolved fluorometer. Adv Biosci Bioeng 4:79. https://doi.org/10.11648 /j.abb.20160406.13

Publisher's Note Springer Nature remains neutral with regard to jurisdictional claims in published maps and institutional affiliations. 